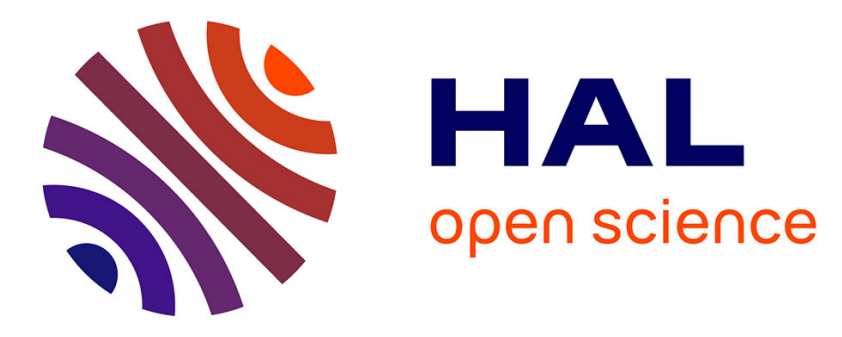

\title{
Winning solutions and post-challenge analyses of the ChaLearn AutoDL challenge 2019
}

Zhengying Liu, Adrien Pavao, Zhen Xu, Sergio Escalera, Fabio Ferreira, Isabelle Guyon, Sirui Hong, Frank Hutter, Rongrong Ji, Julio C S Jacques Junior, et al.

\section{To cite this version:}

Zhengying Liu, Adrien Pavao, Zhen Xu, Sergio Escalera, Fabio Ferreira, et al.. Winning solutions and post-challenge analyses of the ChaLearn AutoDL challenge 2019. 2020. hal-02957135v1

\section{HAL Id: hal-02957135 \\ https://hal.science/hal-02957135v1}

Preprint submitted on 4 Oct 2020 (v1), last revised 8 Jan 2022 (v5)

HAL is a multi-disciplinary open access archive for the deposit and dissemination of scientific research documents, whether they are published or not. The documents may come from teaching and research institutions in France or abroad, or from public or private research centers.
L'archive ouverte pluridisciplinaire HAL, est destinée au dépôt et à la diffusion de documents scientifiques de niveau recherche, publiés ou non, émanant des établissements d'enseignement et de recherche français ou étrangers, des laboratoires publics ou privés. 


\title{
Winning solutions and post-challenge analyses of the ChaLearn AutoDL challenge 2019
}

\author{
Zhengying Liu*, Adrien Pavao*, Zhen Xu*, Sergio Escalera, Fabio Ferreira, Isabelle Guyon, Sirui Hong, \\ Frank Hutter, Rongrong Ji, Julio C. S. Jacques Junior, Ge Li, Marius Lindauer, Zhipeng Luo, Meysam \\ Madadi, Thomas Nierhoff, Kangning Niu, Chunguang Pan, Danny Stoll, Sebastien Treguer, Jin Wang, \\ Peng Wang, Chenglin Wu, Youcheng Xiong, Arbër Zela, Yang Zhang
}

\begin{abstract}
The objective of this research is to push the frontiers in Automated Machine Learning, specifically targeting Deep Learning. We analyse ChaLearn's Automated Deep Learning challenge whose design features include: (i) Code submissions entirely blind-tested, on five classification problems during development, then ten others during final testing. (ii) Raw data from various modalities (image, video, text, speech, tabular data), formatted as tensors. (iii) Emphasis on "any-time learning" strategies by imposing fixed time/memory resources and using the Area under Learning curve as metric. (iv) Baselines provided, including "Baseline 3", combining top-ranked solutions of past rounds (AutoCV, AutoNLP, AutoSpeech,and AutoSeries). (v) No Deep Learning imposed. Principal findings: (1) The top two winners passed all final tests without failure, a significant step towards true automation. Their solutions were open-sourced. (2) Despite our effort to format all datasets uniformly to encourage generic solutions, the participants adopted specific workflows for each modality. (3) Any-time learning was addressed successfully, without sacrificing final performance. (4) Although some solutions improved over Baseline 3, it strongly influenced many. (5) Deep Learning solutions dominated, but Neural Architecture Search was impractical within the time budget imposed. Most solutions relied on fixed-architecture pre-trained networks, with fine-tuning. Ablation studies revealed the importance of meta-learning, ensembling, and efficient data loading, while data-augmentation is not critical.
\end{abstract}

Index Terms-AutoML, Deep Learning, Meta-learning, Neural Architecture Search, Model Selection, Hyperparameter Optimization

\section{INTRODUCTION}

$\mathrm{T}$ HE year of 2019 has seen the success of several machine learning competitions we organized in the Automated Deep Learning (AutoDL) challenge series [1], which provides a reusable benchmark in the domain of Automated Machine Learning (AutoML) applied to Deep Learning. The AutoML problem asks whether one could have one single algorithm (an AutoML algorithm) that can perform learning on a large spectrum of data and always has consistently good performance, removing the need for human expertise (which is exactly the opposite of No Free Lunch theorems [2], [3], [4]). Our AutoDL challenges encompass many domains in which Deep Learning has been success-

*The first three authors contributed equally. The other authors are in alphabetical order of last name. The corresponding author is: Zhengying Liu (zhengying.liu@inria.fr), with Université Paris-Saclay. Adrien Pavao (adrien.pavao@gmail.com) is with Universté Paris-Saclay. Z. Xu (xuzhen@4paradigm.com) is with 4Paradigm, Beijing. S. Escalera is with the Universitat de Barcelona and Computer Vision Center. I. Guyon is with Université Paris-Saclay, INRIA, and ChaLearn. Julio C. S. Jacques Junior is with Universitat Oberta de Catalunya and Computer Vision Center, Spain. M. Madadi is with Computer Vision Center, Spain. Sebastien Treguer is with La Paillase. F. Ferreira, F. Hutter, T. Nierhoff, D. Stoll and A. Zela are with the University of Freiburg, Germany. F. Hutter is also with the Bosch Center for Artificial Intelligence. E-mail: \{ferreira, fh, nierhoff, stolld, zelaa\}@cs.unifreiburg.de. M. Lindauer is with the Leibniz University Hannover, Germany. Peng Wang (wangpeng31@lenovo.com) is with Lenovo AI Lab (upwind flys). E-mail: lindauer@tnt.uni-hannover.de. C.Wu, Y. Zhang, S. Hong and Y. Xiong are with DeepWisdom, China. E-mail: \{alexanderwu, youngzhang, stellahong,ycxiong\}@fuzhi.ai, R. Ji and H. Li are with Xiamen University, China. E-mail: rrji@xmu.edu.cn. Zhipeng Luo, Chunguang Pan, Ge Li, Kangning Niu and Jin Wang are with DeepBlueAI, China. E-mail: \{luozp, panchg, lige, niukn,wangjin \}@deepblueai.com ful: computer vision, natural language processing, speech recognition, as well as classic tabular data (feature-vector representation).

AutoML is crucial to accelerate data science and reduce the need for data scientists and machine learning experts. For this reason, many efforts have been made to achieve true AutoML, both in academia and the private sector. In academia, AutoML challenges [5] have been organized and collocated with top machine learning conferences such as ICML and NeurIPS to motivate AutoML research in the machine learning community. The winning approaches from such prior challenges (e.g. auto-sklearn [6], |7|) are now widely used both in research and in industry. More recently, interest in Neural Architecture Search (NAS) has exploded [8], [9], [10], [11], [12]. On the industry side, many companies such as Microsoft |13| and Google are developing AutoML solutions. Google has also launched various AutoML [14], NAS [6], [15], [16], [17], and meta-learning [18], [19] research efforts. Most of the above approaches, especially those relying on Hyper-Parameter Optimization (HPO) or NAS, require significant computational resources and engineering time to find good models. Additionally, reproducibility is impaired by undocumented heuristics [20].

The motivation behind this AutoDL challenge series is thus two-fold. First, we wish to continue promoting the community's research interests on AutoML to build universal AutoML solutions that can be applied to any task (as long as the data is collected and formatted in the same manner). By choosing tasks in which Deep Learning methods 
excel, we put gentle pressure on the community to improve on Automated Deep Learning. Second, our challenges can serve as reusable benchmarks for fairly evaluating AutoML approaches, on a wide range of domains. Since computational resources and time cost can be a non-negligible factor, we introduce an any-time learning metric called Area under Learning Curve (ALC) (see Section 2.3) for the evaluation of participants' approaches, taking into consideration both the final performance (e.g. accuracy) and the speed to achieve this performance (using wall-time). As far as we know, the AutoDL challenges are the only competitions that adopt a similar any-time learning metric.

Acknowledging the difficulty of engineering universal AutoML solutions, we first organized four preliminary challenges. Each of them focused on a specific application domain. These included: AutoCV for images, AutoCV2 for images and videos, AutoNLP for natural language processing (NLP) and AutoSpeech for speech recognition. Then, during NeurIPS 2019 we launched the final AutoDL challenge that combined all these application domains, and tabular data. All these challenges shared the same competition protocol and evaluation metric (i.e. ALC) and provided data in a similar format. All tasks were multi-label classification problems.

For domain-specific challenges such as AutoCV, AutoCV2, AutoNLP and AutoSpeech, the challenge results and analysis are presented in [1]. In this work, we focus on the final AutoDL challenge with all domains combined together. Some of the principal questions we aimed at answering in this challenge ended up being answered, with the help of fact sheets that participants filled out, and some from the post-challenge experiment, as detailed further in the paper. The main highlights are the briefly summarized.

First of all, were the tasks of the challenge of a difficulty adapted to push the state-of-the-art in Automated Deep Learning? On one hand YES, since (1) the top two ranking participants managed to pass all final tests without code failure and delivered solutions on new tasks (trained and tested without human intervention), performing significantly better than the baseline methods, within the time/memory constraints, and (2) all teams used Deep Learning as part of their solutions. This confirms that Deep Learning is well adapted to the chosen domains (CV, NLP, speech). As further evidence that we hit the right level of challenge duration and difficulty, $90 \%$ of teams found the challenge duration sufficient and $50 \%$ of teams found the time and computational resources sufficient. On the other hand NO, since (1) all of the top-9 teams used a domain-dependent approach, treating each data modality separately (i.e. using hard-coded if..else clauses and will probably fail on new unseen domains such as other sensor data); and (2) the time budget was too constraining to do any Neural Architecture Search; and (3) complex heterogeneous ensembles including non Deep Learning methods were used.

Secondly, was the challenge successful in fostering progress in "any-time learning"? The learning curve examples in Figures 2 and 10a show that for most datasets, convergence was reached within 20 minutes. A fast increase in performance early on in the learning curve demonstrates that the participants made a serious effort to deliver solutions quickly, which is an enormous asset in many applica- tions needing a quick turnover and for users having modest computational resources.

Finally, from the research point of view, a burning question is whether progress was made in "meta-learning", the art of learning from past tasks to perform better on new tasks? There is evidence that the solutions provided by the participants generalize well to new tasks, since they performed well in the final test phase. To attain these results, seven out of the nine top ranking teams reported that they used the provided "public" datasets for meta-learning purposes. In Section 5.1 we used ablation studies to evaluate the importance of using meta-learning and in Section 5.2 we analyzed how well the solutions provided meta-generalize.

Thus, while we are still far from an ultimate AutoML solution that learns from scratch for ALL domains (in the spirit of [17]), we made great strides with this challenge towards democratizing Deep Learning by significantly reducing human effort. The intervention of practitioners is reduced to formatting data in a specified way; we provide code for that at https://autodl.chalearn.org, as well as the code of the winners.

The rest of this work is organized as follows. In Section 2 we give a brief overview of the challenge design (see [21] for detailed introduction). Then, detailed descriptions of winning methods are given in Section 4 Post-challenge analyses, including ablation study results, is presented in Section 5 Lastly, we conclude the work in Section 6

The rest of this work is organized as follows. In Section 2 we give a very brief overview of the challenge design (see [21] for detailed introduction). Then, detailed descriptions of winning methods are given in Section 4 Post-challenge analysis including ablation study results are presented in Section 5 Lastly, we conclude the work in Section 6

\section{Challenge design}

\subsection{Data}

In AutoDL challenges, raw data (images, videos, audio, text, etc) are provided to participants formatted in a uniform tensor manner (namely TFRecords, a standard generic data format used by TensorFlow). For images with native compression formats (e.g. JPEG, BMP, GIF), we directly use the bytes. Our data reader decodes them on-the-fly to obtain a 4D tensor. Video files in mp4/avi format (without the audio track) are used in a similar manner. For text datasets, each example (i.e. a document) is a sequence of integer indices. Each index corresponds to a word (for English) or character (for Chinese) in a vocabulary given in the metadata. For speech datasets, each example is represented by a sequence of floating numbers specifying the amplitude at each timestamp, similar to uncompressed WAV format. Lastly, tabular datasets' feature vector representation can be naturally considered as a special case of our $4 \mathrm{D}$ tensor representation.

For practical reasons, each dataset was kept under 2.5 GB, which required sometimes reducing image resolution, cropping, and/or downsampling videos. We made sure to include application domains in which the scales varied a lot. We formatted around 100 datasets in total and used 66 of them for AutoDL challenges: 17 image, 10 video, 16 text, 16 speech and 7 tabular. The distribution of domain and size 


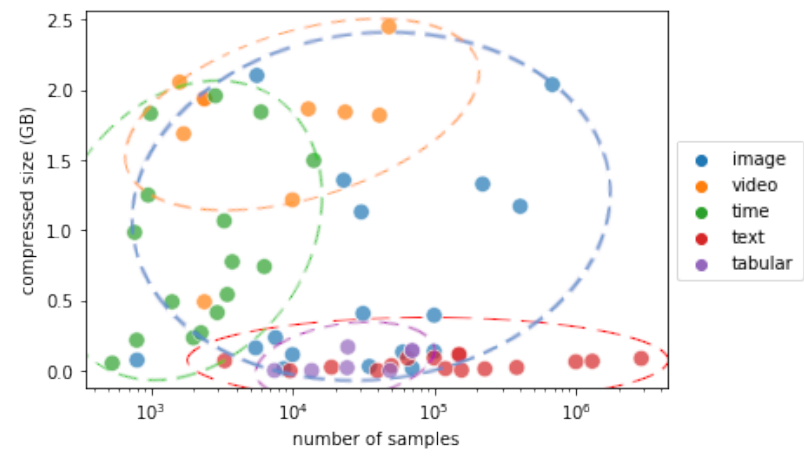

Fig. 1: Distribution of AutoDL challenge dataset domains with respect to compressed storage size in giga-bytes and total number of examples for all 66 AutoDL datasets. We see that the text domain varies a lot in terms of number of examples but remains small in storage size. The image domain varies a lot in both directions. Video datasets are large in storage size in general, without surprise. Speech and time series datasets have fewer number of examples in general. Tabular datasets are concentrated and are small in storage size.

is visualized in Figure 1. All datasets marked public can be downloaded on corresponding challenge websites 1 and information on some meta-features of all AutoDL datasets can be found on the "Benchmark" page $\left.\right|^{2}$ of our website. All tasks are supervised multi-label classification problems, i. e. data samples are provided in pairs $\{X, Y\}, X$ being an input $4 \mathrm{D}$ tensor of shape (time, row, col, channel) and $Y$ a target binary vector (withheld from in test data).

For the datasets of AutoDL challenge, we won't release their identities as we will very probably reuse them in future challenges. But we recall their name, domain and other meta-features in Table 1. These datasets will appear in our analysis frequently.

\subsection{Blind testing}

A hallmark of the AutoDL challenge series is that the code of the participants is blind tested, without any human intervention, in uniform conditions imposing restrictions on training and test time and memory resources, to push the state-of-the-art in automated machine learning. The challenge had 2 phases:

1) A feed-back phase during which methods were trained and tested on the platform on five practice datasets. During the feed-back phase, the participants could make several submissions per day and get immediate feed-back on a leaderboard. The feedback phase lasted 4 months. Obviously, since they made so many submissions, the participants could to some extent get used to the feed-back datasets. For that reason, we also had:

2) A final phase using ten fresh datasets. Only ONE FINAL CODE submission was allowed in that phase.

1. https://autodl.lri.fr/competitions/162

2. https://autodl.chalearn.org/benchmark

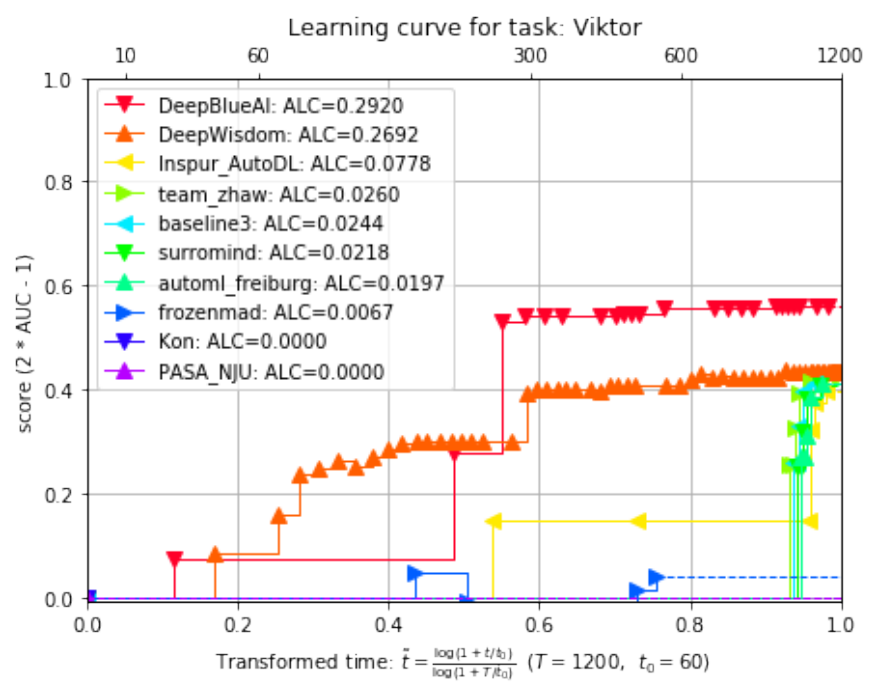

Fig. 2: Learning curves of top-9 teams (together with one baseline) on the text dataset Viktor from the AutoDL challenge final phase. We observe different patterns of learning curves, revealing various strategies adopted by participating teams. The curve of DeepWisdom goes up quickly at the beginning but stabilizes at an inferior final performance (and also inferior any-time performance) than DeepBlueAI. The fact that these two curves cross each other suggests that one might be able to combine these 2 methods to improve the exploration-exploitation trade-off. In terms of number of predictions made during the whole train/predict process (20 minutes), many predictions are made by DeepWisdom and DeepBlueAI but (much) fewer are made by the other teams. Finally, although different patterns are found, some teams such as team_zhaw, surromind and automl_freiburg show very similar patterns. This is because all teams adopted a domain-dependent approach and some teams simply used the code of Baseline 3 for certain domains (text in this case).

Since this was a complete blind evaluation during BOTH phases, we provided additional "public" datasets for practice purposes and to encourage meta-learning.

We ran the challenge on the Codalab platform (http:// competitions.codalab.org), which is an open source project of which we are community lead. Codalab is free for use for all. We use to run the calculations a generous donation of Google of 100,000 cloud units. We prepared a docker including many machine learning toolkits and scientific programming utilities, such as Tensorflow, Pytorch and scikitlearn. We ran the jobs of the participants in virtual machines equipped with NVIDIA Tesla P100 GPUs. One VM was entirely dedicated to the job of one participant during its execution.

\subsection{Metric}

AutoDL challenges encourage any-time learning by scoring participants with the Area under the Learning Curve (ALC) (see definition in Eq. 1. and examples of learning curves can in Figure 2). The participants can train in increments of a chosen duration (not necessarily fixed) to progressively 
TABLE 1: Datasets of the AutoDL challenge, for both phases. The final phase datasets (meta-test datasets) vary a lot in terms of number of classes, number of training examples, and tensor dimension, compared to those in the feedback phase. This was one of the difficulties of the AutoDL challenge. "chnl" codes for channel, "var" for variable size, "CE pair" for "cause-effect pair". More information on all 66 datasets used in AutoDL challenges can be found at https: //autodl.chalearn.org/benchmark

\begin{tabular}{|c|c|c|c|c|c|c|c|c|c|c|c|}
\hline & \multirow{2}{*}{ Dataset } & Phase & Topic & Domain & Class & \multicolumn{3}{|c|}{ Sample number } & \multicolumn{3}{|c|}{ Tensor dimension } \\
num & train & test & time & row & col & chnl \\
\hline \hline 1 & Apollon & feedback & people & image & 100 & 6077 & 1514 & 1 & var & var & 3 \\
\hline 2 & Monica1 & feedback & action & video & 20 & 10380 & 2565 & var & 168 & 168 & 3 \\
\hline 3 & Sahak & feedback & speech & time & 100 & 3008 & 752 & var & 1 & 1 & 1 \\
\hline 4 & Tanak & feedback & english & text & 2 & 42500 & 7501 & var & 1 & 1 & 1 \\
\hline 5 & Barak & feedback & CE pair & tabular & 4 & 21869 & 2430 & 1 & 1 & 270 & 1 \\
\hline 6 & Ray & final & medical & image & 7 & 4492 & 1114 & 1 & 976 & 976 & 3 \\
\hline 7 & Fiona & final & action & video & 6 & 8038 & 1962 & var & var & var & 3 \\
\hline 8 & Oreal & final & speech & time & 3 & 2000 & 264 & var & 1 & 1 & 1 \\
\hline 9 & Tal & final & chinese & text & 15 & 250000 & 132688 & var & 1 & 1 & 1 \\
\hline 10 & Bilal & final & audio & tabular & 20 & 10931 & 2733 & 1 & 1 & 400 & 1 \\
\hline 11 & Cucumber & final & people & image & 100 & 18366 & 4635 & 1 & var & var & 3 \\
\hline 12 & Yolo & final & action & video & 1600 & 836 & 764 & var & var & var & 3 \\
\hline 13 & Marge & final & music & time & 88 & 9301 & 4859 & var & 1 & 1 & 1 \\
\hline 14 & Viktor & final & english & text & 4 & 2605324 & 289803 & var & 1 & 1 & 1 \\
\hline 15 & Carla & final & neural & tabular & 20 & 10931 & 2733 & 1 & 1 & 535 & 1 \\
\hline
\end{tabular}

improve performance, until the time limit is attained. Performance is measured by the NAUC or Normalized Area Under ROC Curve (AUC) $N A U C=2 \times A U C-1$ averaged over all classes. Multi-class classification metrics are not being considered, i.e. each class is scored independently. Since several predictions can be made during the learning process, this allows us to plot learning curves, i. e. "performance" (on test set) as a function of time. Then for each dataset, we compute the Area under Learning Curve (ALC). The time axis is $\log$ scaled (with time transformation in Eq. 2) to put more emphasis on the beginning of the curve. This way, we encourage participants to develop techniques that improve performance rapidly at the beginning of the training process. This should be important to treat large redundant and/or imbalanced datasets and small datasets alike, e. g. by treating effectively redundancy in large training datasets or using learning machines pre-trained on other data if training samples are scarce. Finally, in each phase, an overall rank for the participants is obtained by averaging their ALC ranks obtained on each individual dataset. The average rank in the final phase is used to determine the winners.

\subsection{Baseline 3 of AutoDL challenge}

As in previous challenges (e.g. AutoCV, AutoCV2, AutoNLP and AutoSpeech), we provide 3 baselines (Baseline 0, 1 and 2) for different levels of use: Baseline 0 is just constant predictions for debug purposes, Baseline 1 a linear model, and Baseline 2 a CNN (see [21] for details). In the AutoDL challenge, we provide additionally a Baseline 3 which combines the winning solutions of previous challenges. And for benchmarking purposes, we ran Baseline 3 on all 66 datasets in all AutoDL challenges (public or not) and the results are shown in Figure 3 Many participants used Baseline 3 as a starting point to develop their own method. For this reason, we describe in this section the components of Baseline 3 in some details.

\subsubsection{Vision domain: winning method of AutoCV/AutoCV2}

The wining solution of AutoCV1 and AutoCV2 Challenges [21], i.e., kakaobrain, is based on Fast AutoAugment [22], which is a modified version of the AutoAugment [23] approach. Instead of relying on human expertise, AutoAugment [23] formulates the search for the best augmentation policy as a discrete search problem and uses Reinforcement Learning to find the best policy. The search algorithm is implemented as a Recurrent Neural Network (RNN) controller, which samples an augmentation policy $S$, combining image processing operations, with their probabilities and magnitudes. $S$ is then used to trained a child network to get a validation accuracy $R$, which is used to update the RNN controller by policy gradient methods.

Despite a significant improvement in performance, AutoAugment requires thousands of GPU hours even with a reduced target dataset and small network. On the other hand, Fast AutoAugment [22] finds effective augmentation policies via a more efficient search strategy based on density matching between a pair of train datasets, and a policy exploration based on Bayesian optimization over stratified $k$-folds splits of the training dataset. The winning team (kakaobrain) of AutoCV implemented a light version of Fast AutoAugment, replacing the 5-folds by a single fold search and using a random search instead of Bayesian optimization. The backbone architecture used is ResNet-18 (i.e., ResNet [24] with 18 layers).

\subsubsection{Text domain: winning method of AutoNLP}

For the text domain, Baseline 3 uses the 2nd place's solution from AutoNLP since we found that upwind flys's code was easier to adapt in the challenge setting and gave similar performance to that of 1st place (DeepBlueAI).

The core of upwind flys's solution is a meta-controller dealing with multiple modules in the pipeline including model selection, data preparation and evaluation feedback. For the data preparation step, to compensate for class imbalance in the AutoNLP datasets, upwind_flys first calculates the data distribution of each class in the original data. Then, 
they randomly sample training and validation examples from each class in the training set, thus balancing the training and validation data by up- and down-sampling. Besides, upwind_flys prepares a model pool including fast lightweight models like LinearSVC [25], and heavy but more accurate models like LSTM [26] and BERT [27]. They first use light models (such as linear SVC), but the metacontroller switches eventually to other models such as neural networks, with iterative training. If the AUC drops below a threshold or drops twice in a row, the model is switched, or the process is terminated and the best model ever trained is chosen, when the pool is exhausted.

\subsubsection{Speech domain: winning method of AutoSpeech}

Baseline 3 uses the approach of the 1st place winner of the AutoSpeech challenge: PASA_NJU. Interestingly, PASA_NJU, has developed one single approach for the two sequence types of data, i.e. speech and text. As time management is key for optimizing any time performance, as measured by the metric derived from the ALC, the best teams have experimented with various data selection and progressive data loading approaches. Such decision allowed them to create a tread-off between accelerating the first predictions while ensuring a good and stable final AUC. For instance PASA_NJU truncated speech samples from $22.5 \mathrm{~s}$ to $2.5 \mathrm{~s}$, and started with loading $50 \%$ of the samples for the 3 first training loops, however preserving a similar balance of classes, loading the rest of the data from the 4th training loop. As for features extraction, MFCC (MelFrequency Cepstral Coefficients) [28] and STFT (Short-Time Fourrier Transform) [29] are used.. In terms of model selection and architectures, PASA_NJU progressively increases the complexity of their model, starting with simple models like LR (Logistic Regression), LightGBM at the beginning of the training, combined later with some light weight pretrained CNN models like Thin-ResNet-34 (ResNet [24] but with smaller numbers of filters/channels/kernels) and VggVox [30], finally (bidirectional) LSTM |26|, with attention mechanism. This strategy allows to make fast early predictions and progressively improves models performance over time to optimize the anytime performance metric.

\subsubsection{Tabular domain}

As there were no previous challenge for the tabular domain in AutoDL challenge series, the organizers implemented a simple multi-layer perceptron (MLP) baseline. Tabular datasets consist of both continuous values and categories. Categorical quantities are converted to normalized indices. Tabular domains may have missing values (missing values are replaced by zero) as well. Therefore, to cope with missing data, we designed a denoising autoencoder (DAE) [31] able to interpolate missing values from available data. The architecture consists of a batch normalization layer right after input data, a dropout, 4 fully connected (FC) layers, a skip connection from the first FC layer to the 3rd layer and an additional dropout after 2nd FC layer. Then we apply a MLP classifier with 5 FC layers. All FC layers have 256 nodes (expect the last layers of DAE and classifier) with ReLU activation and batch normalization. We keep the same architecture for all datasets in this domain. DAE loss is a
L1 loss on non-missing data and classifier loss is a sigmoid cross entropy.

\section{AutoDl ChaLLENGE RESULtS}

The AutoDL challenge (the last challenge in the AutoDL challenges series 2019) lasted from 14 Dec 2019 (launched during NeurIPS 2019) to 3 Apr 2020. It has had a participation of 54 teams with 247 submissions in total and 2614 dataset-wise submissions. Among these teams, 19 of them managed to get a better performance (i.e. average rank over the 5 feedback phase datasets) than that of Baseline 3 in feedback phase and entered the final phase of blind test. According to our challenge rules, only teams that provided a description of their approach (by filling out some fact sheets we sent out) were eligible for getting a ranking in the final phase. We received 8 copies of these fact sheets and thus only these 8 teams were ranked. These teams are (alphabetical order): DeepBlueAI, DeepWisdom, frozenmad, Inspur_AutoDL, Kon, PASA_NJU, surromind, team_zhaw. One team (automl_freiburg) made a late submission and isn't eligible for prizes but will be included in the post-analysis for scientific purpose.

The final ranking is computed from the performances on the 10 unseen datasets in the final phase. To reduce the variance from diverse factors such as randomness in the submission code and randomness of the execution environment (which makes the exact ALC scores very hard to reproduce since the wall-time is hard to control exactly), we re-run every submission several times and average the ALC scores. The average ALC scores obtained by each team is shown in Figure 4 (the teams are ordered by their final ranking). From this figure, we see that some entries failed constantly on some datasets such as frozenmad on Yolo, Kon on Marge and PASA_NJU on Viktor, due to issues in their code (e.g. bad prediction shape or out of memory error). On the other hand, some entries crashed only sometimes on certain datasets, such as Inspur_AutoDL on Tal, whose cause is related to some pre-processing procedure on text datasets concerning stop words. Otherwise, the error bars show that the performances of most runs are statistically consistent.

\section{WiNNING APPROACHES}

In this section, we present in detail the winning solutions from top-3 winning teams (DeepWisdom, DeepBlueAI and PASA_NJU) and the team automl_freiburg which made a late submission in feedback phase but ranked 5 th in final phase. We considered interesting to introduce automl_freiburg's approach due to their contributions and for scientific purpose.

A summary of the winning approaches on each domain can be found in Table 2 Another summary using a categorization by machine learning techniques can be found in Table 3. We see in Table 2 that almost all approaches used 5 different methods from 5 domains. For each domain, the winning teams' approaches are much inspired by Baseline 3. In Table 3, we see that almost all different machine learning techniques are actively present and frequently used in all domains (exception some rare cases for example transfer learning on tabular data). We'll introduce below in detail the top-3 winning solutions. 


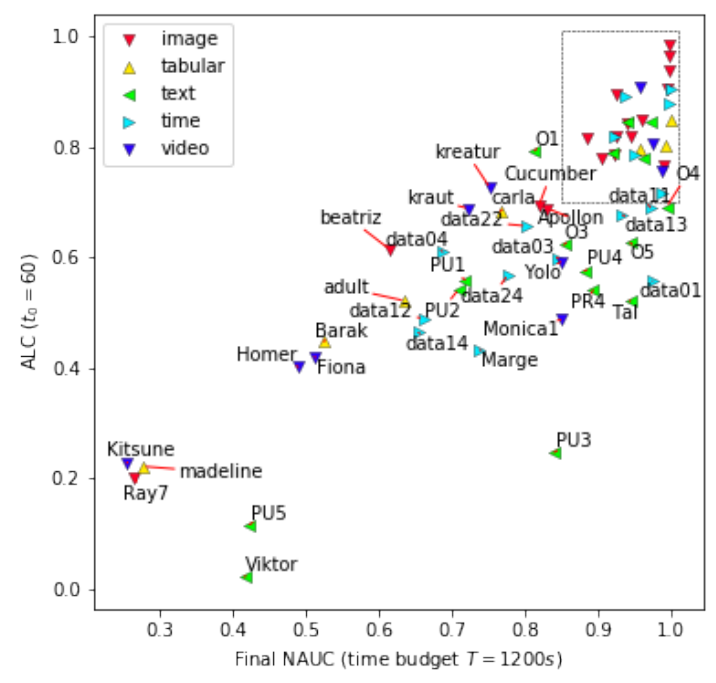

(a) All results included

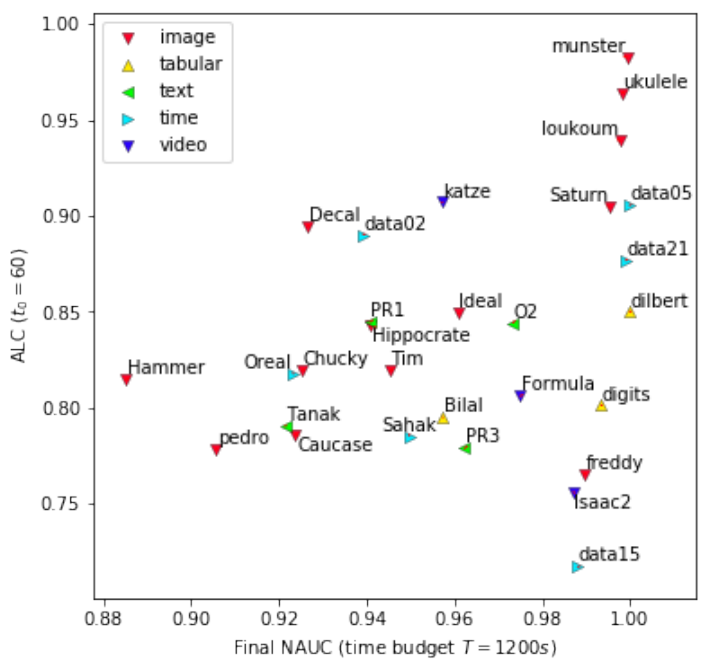

(b) Rectangular area in Figure 3a zoomed

Fig. 3: ALC and final NAUC performances of Baseline 3 on ALL 66 AutoDL datasets. Different domains are shown with different markers. In 3a the dataset name is shown beside each point except the top-right area, which is shown in Figure $3 \mathrm{~b}$ These figures will serve as a reference to compare future methods with Baseline 3. Numerical values are provided in appendix in Table 4

\subsection{Approach of DeepWisdom (1st prize)}

This team proposed a unified learning framework following a meta-learning paradigm. The framework consists of two parts: meta-train and meta-inference. The meta-train module takes as input the "public" datasets, which are augmented by the internal data augmentation engine, and the objective function (the ALC metric in the case of the challenge). The meta-trainer generates solution agents, whose objective is to search for best models, using search operators. In the meta-inference step, a new task is processed taking in one dataset of the challenge. Initial metadata and seed data (few-shot samples) are acquired from the raw dataset.This constitutes the input of the solution agents obtained by metatraining. Solution workflow starts after taking in the seed input data, then it receives more raw data in a streaming way, and interacts with a whole set of tables for storage to cache intermediate results and models. Next, we explain the domain-specific contributions of this team.

In the image domain, ResNet-18 is used in the early stages of the training and then switched to ResNet-9 in more advanced stages (The reason is the instability of ResNet-18). When switching from ResNet-18 to ResNet-9, to reduce I/O cost, they cache the mini batches, which have been used for ResNet-18 training in GPU and reuse them for the initial training phase of ResNet-9, until all these mini batches are exhausted. The networks are fine-tuned by initialing from Imagenet pre-trained networks. However, for a fast transfer learning batch normalization and bias variables are initialized from scratch. To avoid overfitting, fast auto augmentation is used in the later training phase, which can automatically search for the best augmentation strategy on the given dataset, according to the validation AUC. The searching process is quite time-consuming but effectively increase the top-AUC.

In the video domain, a mixed convolution (MC3) net- work [39] is adopted which consists of 3D convolutions in the early layers and 2D convolutions in the top layers of the network. The network is pretrained on the Kinetics dataset and accelerated transferring to other datasets by reinitializing linear weights and bias and freezing the first two layers. Due to the slower speed of 3D than 2D convolution, 3 frames are extracted at the early phase. Then for longer videos, an ensemble strategy is applied to combine best predictions from MC3 with 3-,10- and 12-frames data.

In the speech domain, a model search is applied in the meta-training part and LogisticRegression and ThinResnet34 achieve best performance in non-neural and neural models, respectively. The meta-trainer firstly learned that validating in the beginning was wasting the time budget without any effect on ALC, thus the evaluation agent did not validate when model was fitting new streaming data. Secondly, if amount of training samples was not very large, evaluation metric on training data could avoid overfitting partly while last best predictions ensemble strategy was applied.

In the text domain, they decode maximum 5500 samples for each round. Various data preprocessing methods are applied, including email data structure pre-processing, word frequency filtering and word segmentation. After tokenization and sequence padding, both pre-trained and randomly initialized word embedding (with various dimensions) are used as word features. The meta-trainer includes several solutions such as TextCNN, RCNN, GRU, and GRU with attention [40], [41]. Hyperparameters are set after a neural network architecture is selected. Also a weighted ensembling is adopted among top 20 models based AUC scores.

Finally, in the tabular domain, they batch the dataset and convert tfdatasets to numpy format progressively, a weighted ensembling is applied based on several optimized models including LightGBM, Catboost, Xgboost and DNN 


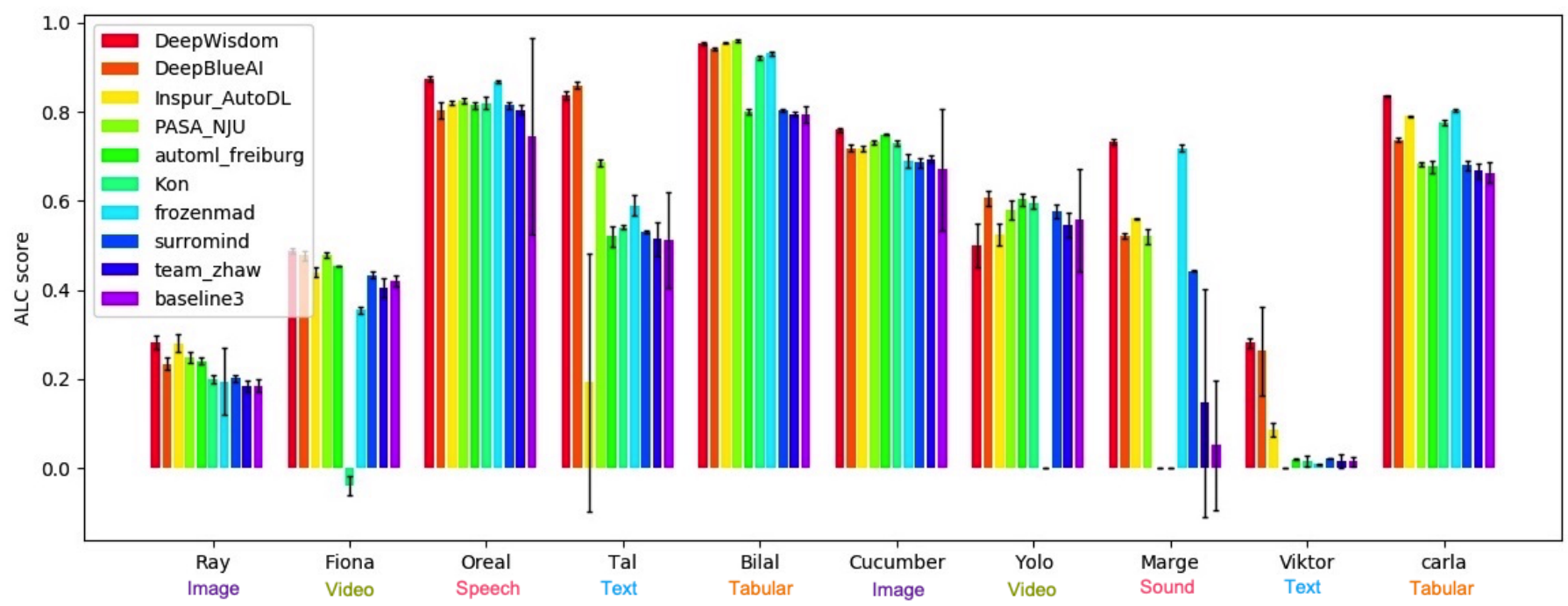

Fig. 4: ALC scores of top 9 teams in AutoDL final phase averaged over repeated evaluations (and Baseline 3, for comparison). The entry of top 6 teams are re-run 9 times and 3 times for other teams. Error bars are shown with (half) length corresponding to the standard deviation from these runs. Some (very rare) entries are excluded for computing these statistics due to failures caused by the challenge platform backend. The team ordering follows that of their average rank in the final phase. The domains of the 10 tasks are image, video, speech/times series, text, tabular (and then another cycle in this order). More information on the task can be found in Table 1 .

on the offline datasets. To do so, data is split to several folds. Each fold has a training set and two validation sets. One validation set is used to optimize model hyperparameters and other set to compute ensembling weights.

\subsection{Approach of DeepBlueAl (2nd prize)}

The DeepBlueAI solution is a combination of methods that are specific to each modality. Nevertheless, three concepts are applied across all modalities: 1) optimizing time budget by reducing the time for data processing, start with light models and parameters setting to accelerate first predictions; 2) dataset adaptive strategies and 3) ensemble learning.

For images, the DeepBlueAI team applies a strategy adapted to each specific dataset. They apply a pretrained ResNet-18 model. The dataset adaptive strategy is not applied to model selection but to parameters settings including: image size, steps per epoch, epoch after which starting validating and fusing results. With the aim to optimize for final AUC, and make results more stable, they apply a progressive ensemble learning method, i.e. for epochs between 5 to 10 , the latest 2 predictions are averaged, while after 10 epochs the 5 latest predictions are averaged. When the score on validation set improves a little, data augmentation strategy is adopted by searching for the most suitable data augmentation strategy for each image dataset with a small scale version of Fast AutoAugment [42] limiting the search among 20 iterations in order to preserve more time for training.

For video, ResNet-18 is used for classification. In the search for a good trade-off between calculation speed and classification accuracy, 1/6 of the frames with respect to the total number are selected. For datasets with a large number of categories, image size is increased to 128 to get more details out of it. During training, when the score of the validation set increases, predictions are made on the test set, and submitted as the average of the current highest 5 test results.

For speech, features are extracted with Mel spectrogram [43] for Logistic Regression (LR) model and MFCC [28] for deep learning models. In order to accelerate the extraction long sequences are truncated but covering at least $90 \%$ of the sequence. Then, to accelerate first score computation, training data are loaded progressively, $7 \%$ for the first iteration, then $28 \%, 66 \%$ and then all data at 4 th iteration, with care to balance multiple categories, to ensure the models can learn accurately. As for the models, LR is used for the first 3 iterations, then from the 4 th iteration using all the data deep learning models, CNN and CNN+GRU [44] are employed. At the end, the overall 5 best models and the best version of each of the 3 models are averaged to build a final ensemble. The iterative data loading is especially effective on large dataset and play a significant role in the performance measured by the metric derived from the ALC.

For text, the data set size, text length and other characteristics are automatically obtained, and then a preprocessing method suitable for the data set is adopted. Long texts, over 6000 words are truncated, and NLTK stemmer is used to extract root features and filter meaningless words with frequency below 3. As for model selection, FastText [45], TextCNN |40], BiGRU [44| are used by their system that generate different model structures and set of parameters adapted to each dataset. The size of the data set, the number of categories, the length of the text, and whether the categories are balanced are considered to generate the most suitable models and parameter settings.

For tabular, three directions are optimized: accelerating scoring time, adaptive parameter setting, ensemble learning.

Data is first split into many batches to significantly 
TABLE 2: Summary of the five top ranking solutions and their average rank in the final phase. The participant's average rank (over all tasks) in the final phase is shown in parenthesis (automl_freibug and Baseline 3 were not ranked in the challenge). Each entry concerns the algorithm used for each domain and is of the form "[pre-processing / data augmentation]-[transfer learning/meta-learning]-[model/architecture]-[optimizer]" (when applicable).

\begin{tabular}{|c|c|c|c|c|c|}
\hline Team & image & video & speech & text & tabular \\
\hline $\begin{array}{l}\text { 1.DeepWisdom } \\
\text { (1.8) }\end{array}$ & $\begin{array}{c}\text { [ResNet-18 and ResNet-9 } \\
\text { models] [pretrained on } \\
\text { ImageNet] }\end{array}$ & $\begin{array}{c}\text { [MC3 model] [pretrained } \\
\text { on Kinetics] }\end{array}$ & $\begin{array}{c}\text { [fewshot learning ] [LR, } \\
\text { Thin ResNet34 models] } \\
\text { [pretrained on VoxCeleb2] }\end{array}$ & $\begin{array}{l}\text { [fewshot learning] [task } \\
\text { difficulty and similarity } \\
\text { evaluation for model } \\
\text { selection] [SVM, } \\
\text { TextCNN,[fewshot } \\
\text { learning] RCNN, GRU, } \\
\text { GRU with Attention] }\end{array}$ & $\begin{array}{c}\text { [LightGBM, Xgboost, } \\
\text { Catboost, DNN models] } \\
\text { [no pretrained] }\end{array}$ \\
\hline $\begin{array}{l}\text { 2.DeepBlueAI } \\
\text { (3.5) }\end{array}$ & $\begin{array}{l}\text { [data augmentation with } \\
\text { Fast AutoAugment] } \\
\text { [ResNet-18 model] }\end{array}$ & $\begin{array}{c}\text { [subsampling keeping } 1 / 6 \\
\text { frames] [Fusion of } 2 \text { best } \\
\text { models ] }\end{array}$ & $\begin{array}{c}\text { [iterative data loader }(7, \\
28,66,90 \%) \text { ] [MFCC and } \\
\text { Mel Spectrogram } \\
\text { preprocessing] [LR, CNN, } \\
\text { CNN+GRU models] }\end{array}$ & $\begin{array}{c}\text { [Samples truncation and } \\
\text { meaningless words } \\
\text { filtering] [Fasttext, } \\
\text { TextCNN, BiGRU models] } \\
\text { [Ensemble with restrictive } \\
\text { linear model] }\end{array}$ & $\begin{array}{c}\text { [3 lightGBM models] } \\
\text { [Ensemble with Bagging] }\end{array}$ \\
\hline$\underset{(4)}{\text { 3.Inspur_AutoDL }}$ & \multicolumn{4}{|c|}{ Tuned version of Baseline 3} & $\begin{array}{c}\text { [Incremental data loading } \\
\quad \text { and train- } \\
\text { ing][HyperOpt][LightGBM] }\end{array}$ \\
\hline 4.PASA_NJU (4.1) & $\begin{array}{c}\text { [shape standardization and } \\
\text { image flip (data } \\
\text { augmentation)][ResNet-18 } \\
\text { and SeResnext50] }\end{array}$ & $\begin{array}{c}\text { [shape standardization and } \\
\text { image flip (data } \\
\text { augmentation)][ResNet-18 } \\
\text { and SeResnext50] }\end{array}$ & $\begin{array}{c}\text { [data truncation(2.5s to } \\
22.5 \mathrm{~s})][\text { LSTM, VggVox } \\
\text { ResNet with pretrained } \\
\text { weights of DeepWis- } \\
\text { dom(AutoSpeech2019) } \\
\text { Thin-ResNet34] }\end{array}$ & $\begin{array}{l}\text { [data truncation(300 to } \\
1600 \text { words)][TF-IDF and } \\
\text { word embedding] }\end{array}$ & $\begin{array}{c}\text { [iterative data loading] } \\
\text { [Non Neural Nets models] } \\
\text { [models complexity } \\
\text { increasing over time] } \\
\text { [Bayesian Optimization of } \\
\text { hyperparameters] }\end{array}$ \\
\hline 5.frozenmad (5) & $\begin{array}{c}\text { [images resized under } \\
\text { 128x128] [progressive data } \\
\text { loading increasing over } \\
\text { time and epochs] } \\
\text { [ResNet-18 model] } \\
\text { [pretrained on ImageNet] }\end{array}$ & $\begin{array}{c}\text { [Successive frames } \\
\text { difference as input of the } \\
\text { model] [pretrained } \\
\text { ResNet-18 with RNN } \\
\text { models] }\end{array}$ & $\begin{array}{c}\text { [progressive data loading } \\
\text { in } 3 \text { steps } 0.01,0.4,0.7] \\
\text { [time length adjustment } \\
\text { with repeating and } \\
\text { clipping] [STFT and Mel } \\
\text { Spectrogram } \\
\text { preprocessing] [LR, } \\
\text { LightGBM, VggVox } \\
\text { models] }\end{array}$ & $\begin{array}{c}\text { [TF-IDF and BERT } \\
\text { tokenizers] [ SVM, } \\
\text { RandomForest , CNN, } \\
\text { tinyBERT ] }\end{array}$ & $\begin{array}{c}\text { [progressive data loading] } \\
\text { [no preprocessing] [Vanilla } \\
\text { Decision Tree, } \\
\text { RandomForest, Gradient } \\
\text { Boosting models applied } \\
\text { sequentially over time] }\end{array}$ \\
\hline automl_freiburg & $\begin{array}{r}\text { Architecture and hyperpa } \\
\text { meta-training tasks with } \\
\text { unseen meta-test tasks } \\
\text { EfficientNet [pretrained or } \\
\text { ResNet-18 [KakaoBrain } \\
\text { Forest, Logis }\end{array}$ & $\begin{array}{l}\text { rameters learned offline on } \\
\text { 3OHB. Transfer-learning on } \\
\text { with AutoFolio. Models: } \\
\text { a ImageNet with AdvProp], } \\
\text { weights], SVM, Random } \\
\text { tic Regression }\end{array}$ & \multicolumn{3}{|c|}{ Baseline 3} \\
\hline Baseline 3 & \begin{tabular}{|} 
[Data augmentation with \\
Fast AutoAugment, \\
adaptive input \\
size][Pretrained on \\
ImageNet][ResNet- \\
18(selected \\
offline)]
\end{tabular} & $\begin{array}{c}\text { [Data augmentation with } \\
\text { Fast AutoAugment, } \\
\text { adaptive input size, } \\
\text { sample first few frames, } \\
\text { apply stem CNN to reduce } \\
\text { to } 3 \text { channels][Pretrained } \\
\text { on ImageNet][ResNet- } \\
18(\text { selected } \\
\text { offline)] }\end{array}$ & $\begin{array}{c}\text { [MFCC/STFT feature][LR, } \\
\text { LightGBM, } \\
\text { Thin-ResNet-34, VggVox, } \\
\text { LSTM] }\end{array}$ & $\begin{array}{l}\text { [resampling training } \\
\text { examples][LinearSVC, } \\
\text { LSTM, BERT] }\end{array}$ & $\begin{array}{c}\text { [interpolate missing } \\
\text { value][MLP of four hidden } \\
\text { layers] }\end{array}$ \\
\hline
\end{tabular}

accelerate the data loading and converted from TFrecords to numpy format. In terms of models, decision trees LightGBM are adopted to get faster scoring than with deep learning models. Because LightGBM supports continuous training, and the model learns faster in the early stage. During the training phase, earnings from the previous epochs are much higher than those from the latter. Therefore, a complete training is intelligently divided into multiple parts. The result is submitted after each part to get score faster.

In terms of adaptive parameter setting, some parameters are automatically set according to the size of data and the number of features of the tables. If the number of samples is relatively large, the ensemble fraction is reduced. If the original features of the sample are relatively large, the feature fraction is reduced. A learning rate decay is applied, starting with a large value to ensure a speed up in the early training. An automatic test frequency is adopted. Specifically, the frequency of testing is controlled based on training speed and testing speed. If the training is slow and the prediction is fast, the frequency of the test is increased. On the contrary, if training is fast and prediction is slow, the frequency is reduced. This strategy can improve to higher early scores.

In order to improve generalization, multiple lightGBM models are used to make an ensemble with a bagging method.

\subsection{Approach of PASA_NJU (3rd prize)}

The PASA_NJU team modeled the problem as three different tasks: CV (image and video), Sequence (speech and text) and Tabular (tabular domain).

For the CV task, they preprocessed the data by analysing few sample instances of each dataset at training stage (such as image size, number of classes, video length, etc) in order to standardize the input shape of their model. Then, simple transformations (image flip) were used to augment the data. Random frames were obtained from video files and treated as image database. For both Image and Video tasks, ResNet18 [24] is used. However, SeResnext50 [46] was used at later stages. Basically, they monitor the accuracy obtained by the ResNet-18 model and change the model to the SeResnext50 if no significant improvement is observed. 
TABLE 3: Machine learning techniques applied to each of the 5 domains considered in AutoDL challenge.

\begin{tabular}{|c|c|c|c|c|c|}
\hline ML technique & image & video & speech & text & tabular \\
\hline Meta-learning & \multicolumn{5}{|c|}{$\begin{array}{c}\text { Offline meta-training transferred with AutoFolio }[\overline{32} \text { based on meta-features (automl_freiburg, for image and video) } \\
\text { Offline meta-training generating solution agents, searching for optimal sub-operators in predefined sub-spaces, based on dataset meta-data. } \\
\text { (DeepWisdom) } \\
\text { MAML-like method [18 (team_zhaw) }\end{array}$} \\
\hline $\begin{array}{l}\text { Hyperparameter } \\
\text { Optimization }\end{array}$ & \multicolumn{2}{|c|}{$\begin{array}{l}\text { Offline with BOHB } 33 \text { (Bayesian Optimization and } \\
\text { Multi-armed Bandit) (automl freiburg) Sequential } \\
\text { Model-Based Optimization for General Algorithm } \\
\text { Configuration (SMAC) [34 (automl_freiburg) }\end{array}$} & $\begin{array}{l}\text { Online model complexity } \\
\text { adaptation (PASA_NJU) }\end{array}$ & $\begin{array}{c}\text { Online model selection } \\
\text { and early stopping using } \\
\text { validation set (Baseline 3( } \\
\text { _flys)) }\end{array}$ & $\begin{array}{l}\text { Bayesian Optimization } \\
\text { (PASANJU) } \\
\text { HyperOpt [35] } \\
\text { (Inspur_AutoDL) }\end{array}$ \\
\hline Transfer learning & $\begin{array}{c}\text { Pre-trained on } \\
\text { ImageNet } 36 \text { (all teams } \\
\text { except Kon) }\end{array}$ & $\begin{array}{l}\text { Pre-trained on } \\
\text { ImageNet }[36 \text { (all top-8 } \\
\text { teams except Kon) } \\
\text { MC3 model pretrained on } \\
\text { Kinetics (DeepWisdom) }\end{array}$ & $\begin{array}{l}\text { ThinResnet34 pre-trained } \\
\text { on VoxCeleb2 } \\
\text { (DeepWisdom) }\end{array}$ & $\begin{array}{l}\text { BERT-like } 27 \text { models } \\
\text { pretrained on FastText }\end{array}$ & (not applicable) \\
\hline $\begin{array}{l}\text { Ensemble } \\
\text { learning }\end{array}$ & $\begin{array}{c}\text { Adaptive Ensemble } \\
\text { Learning (ensemble latest } \\
2 \text { to } 5 \text { predictions) } \\
\text { (DeepBlueAI) }\end{array}$ & $\begin{array}{c}\text { Ensemble Selection 37 } \\
\text { (top 5 validation } \\
\text { predictions are fused) } \\
\text { (DeepBlueAI); Ensemble } \\
\text { models sampling 3, 10, } 12 \\
\text { frames (DeepBlueA) }\end{array}$ & $\begin{array}{l}\text { last best predictions } \\
\text { ensemble strategy } \\
\text { (DeepWisdom) } \\
\text { averaging } 5 \text { best overall } \\
\text { and best of each model: } \\
\text { LR, CNN, CNN+GRU } \\
\text { (DeepBlueA) }\end{array}$ & $\begin{array}{l}\text { Weighted Ensemble over } \\
20 \text { best models [37] } \\
\text { (DeepWisdom) }\end{array}$ & $\begin{array}{l}\text { LightGBM ensemble with } \\
\text { bagging method [38] } \\
\text { (DeepBlueAI), } \\
\text { Stacking and blending } \\
\text { (DeepWisdom) }\end{array}$ \\
\hline
\end{tabular}

Speech and Text data are treated similarly, i.e., as a Sequence task. In a preprocessing stage, data samples are cut to have the same shape. Their strategy was to increase the data length as time pass. For example, they use raw data from $2.5 \mathrm{~s}$ to $22.5 \mathrm{~s}$ in speech task, and from 300 to 1600 words when Text data is considered. In both cases, hand-crafted feature extraction methods are employed. For speech data, mel spectrogram, MFCC and STFT [29] is used. When Text is considered, TF-IDF and word embedding is used. To model the problem, they employed Logistic Regression at the first stages and use more advanced Neural Networks at later stages, such as LSTM and Vggvox Resnet [30] (for speech data), without any hyperparameter optimization method. In the case of Vggvox Resnet, pretrained model from Deepwisdom's team from AutoSpeech Challenge 2019 [1] was used.

For Tabular data, they divided the entire process into three stages based on the given time budget, named Retrieve, Feature, and Model, and employed different models and data preprocessing methods at each stage, aiming to have quick responses at early stages. The main task of the Retrieve stage is to get the data and predict as soon as possible. Each time a certain amount of data is acquired, a model is trained using all the acquired data. Thus, the complexity of the model is designed to increase with time. The main task of the Feature stage is to search for good features. As the Neural Feature Seacher(NFS) [47] method uses RNN as the controller to generate the feature sequence, they used the same method and speed up the process by parallelizing it. Finally, at the Model stage, the goal is to search for a good model and hyperparameters. For this, they use hyperopt [48], which is an open-source package that uses Bayesian optimization to guide the search of hyperparameters.

\subsection{Approach of automl_freiburg}

In contrast to other teams, automl freiburg adopts a domainindependent approach but focused only the computer vision tasks (i.e. image and video datasets) of this challenge. While for all other tasks automl_freiburg simply submitted the baseline to obtain the baseline results, they achieved significant improvement on the computer vision tasks w.r.t. the baseline method. To improve both efficiency and flexibility of the approach, they first exposed relevant hyperparameters of the previous AutoCV/AutoCV2 winner code [49] and identified well-performing hyperparameter configurations on various datasets through hyperparameter optimization with BOHB [33]. They then trained a cost-sensitive metamodel [50] with AutoFolio [32] - performing hyperparameter optimization for the meta-learner - that allows to automatically and efficiently select a hyperparameter configuration for a given task based on dataset meta-features. The proposed approach on the CV task is detailed next.

First, they exposed important hyperparameters of the AutoCV/AutoCV2 winner's code [49] such as the learning rate, weight decay or batch sizes. Additionally, they exposed hyperparameters for the online execution (which were hardcoded in previous winner solution) that control, for example, when to evaluate during the submission and the number of samples used. To further increase the potential of the existing solution, they extended the configuration space to also include:

- An EfficientNet [51] (in addition to kakaobrain's [49] ResNet-18) pre-trained on ImageNet [36];

- The proportion of weights frozen when fine-tuning;

- Additional stochastic optimizers (Adam [52], AdamW [53], Nesterov accelerated gradient [54]) and learning rate schedules (plateau, cosine [55|);

- A simple classifier (either a SVM, random forest or logistic regression) that can be trained and used within the first 90 seconds of the submission.

After the extension of the configuration space, they optimized the hyperparameters with BOHB [33] across 300 evaluation runs with a time budget of 300 seconds on eight different datasets (Chucky [56], Hammer [57], Munster [58], caltech_birds2010 [59], cifar100 [56], cifar10 [56], colorectal_histology [60] and eurosat [61]). These eight datasets were chosen from meta-training data to lead to a portfolio of complementary configurations [62], [63]. Additionally, they added a robust configuration to the portfolio of con- 
figurations that performed best on average across the eight datasets. Then, they evaluated each configuration of the portfolio for 600 seconds on all 21 image datasets they had collected. In addition, they searched for a tenth configuration (again with $\mathrm{BOHB}$ ), called the generalist, that they optimized for the average improvement across all datasets relative to the already observed ALC scores. In the end, the meta-train-data consisted of the ALC performance matrix (portfolio configurations $\times$ datasets) and the meta-features from the 21 datasets. These meta-features consisted of the image resolution, number of classes, number of training and test samples and the sequence length (number of video frames, i.e. 1 for image datasets). In addition, they studied the importance of the meta features for the meta-learner, and selected an appropriate subset. To optimize the portfolio further, they applied a greedy submodular optimization [63], |64 to minimize the chance of wrong predictions in the online phase. Based on this data, they trained a cost-sensitive meta-model [50] with AutoFolio [32], which applies algorithm configuration based on SMAC |34|, |65| to efficiently optimize the hyperparameters of the meta-learner. Since the meta-learning dataset was rather small, HPO for the metalearner could be done within a few seconds. Lastly, they deployed the learned AutoFolio model and the identified configurations into the initialization function of the winner's solution code. The workflow of this approach is shown in Figure 5

\section{Post-challenge analyses}

\subsection{Ablation study}

To analyze the contribution of different components in each winning team's solution, we asked 3 teams (DeepWisdom, DeepBlueAI and automl_freiburg) to carry out an ablation study, by removing or disabling certain component (e.g. meta-learning, data augmentation) of their approach. We will introduce in the following more details on these ablation studies by team and synthesize thereafter.

\subsubsection{DeepWisdom}

According to the team DeepWisdom, three of the most important components leading to the success of their approach are: meta-learning, data loading and data augmentation. For the ablation study, these components are removed or disabled in the following manner:

- Meta-learning (ML): Here meta-learning includes transfer learning, pretrain models, and hyperparameter setting and selection. Meta learning is crucial to both the final accuracy performance and the speed of train-predict lifecycle. For comparison we train models from scratch instead of loading pretrained models for image, video and speech data, and use the default hyperparameter settings for text and tabular subtasks.

- Data Loading (DL): Data loading is a key factor in speeding up training procedures to achieve higher ALC score. We improve data loading in several aspects. Firstly, we can accelerate decoding the raw data formatted in a uniform tensor manner to numpy formats in a progressive way, and batching the dataset for text and tabular data could make the conversion faster. Secondly, the cache mechanism is utilized in different levels of data and feature management, and thirdly, video frames are extracted in a progressive manner.

- Data Augmentation (DA): Fast auto augmentation, time augmentation and a stagewise spec_len configuration for thinresnet34 model are considered as data augmentation techniques for image, video and speech data respectively.

We carried out experiments on the 10 final phase datasets with above components removed. The obtain ed ALC scores are presented in Figure 6. As it can be seen in Figure 6 Meta-Learning can be considered one of the most important single component in DeepWisdom's solution. Pre-trained models contribute significantly to both accelerating model training and obtaining higher AUC scores for image, video and speech data, and text and tabular subtasks benefit from hyperparameter setting such as model settings and learning rate strategies. For image, we remove pretrained models for both ResNet-18 and ResNet-9, which are trained on the ImageNet dataset with $70 \%$ and $65 \%$ top 1 test accuracy; for video, we remove the parts of freezing and refreezing the first two layers. Then the number of the frames for ensemble models and replace MC3 model with ResNet-18 model. For speech, we do not load the pre-trained model which is pre-trained on VoxCeleb2 dataset, that is we train the thinresnet34 model from scratch. For text, we use default setting, i.e. do not perform meta strategy for model selections and do not perform learning rate decay strategy selections. For tabular, with the experience of datasets inside and outside this competition, we found two sets of params of lightgbm. The first hyperparameters focus on the speed of lightgbm trainning, it use smaller boost round and max depth, bigger learning rates and so on. While the second hyperparameters focus on the effect of lightgbm trainning, it can give us a generally better score. We use the default hyperparameters in lightgbm in the minus version.

Data Loading is a salient component for the ALC metric in any-time learning. For text, speech and tabular data, data loading speeds up numpy data conversion to make the first several predictions as quickly as possible, achieving higher alc scores. In the minus version, we convert all train tfdatasets to numpy array in the first round, and alc scores of nearly all datasets on all modalities decrease steadily compared with full version solution.

The data augmentation component also helps the alc scores of several datasets. In the minus version for speech data we use the fixed spec_len config, the default value is 200. Comparison on Marge and Oreal datasets is obvious, indicating that longer speech signal sequences could offer more useful information. Fast auto augmentation and test time augmentation enhance performance on image and video data marginally.

\subsubsection{DeepBlueAl}

According to the team DeepBlueAI, three of the most important components leading to the success of their approach are: adaptive strategies, ensemble learning and scoring time reduction. For the ablation study, these components are removed or disabled in the following manner: 


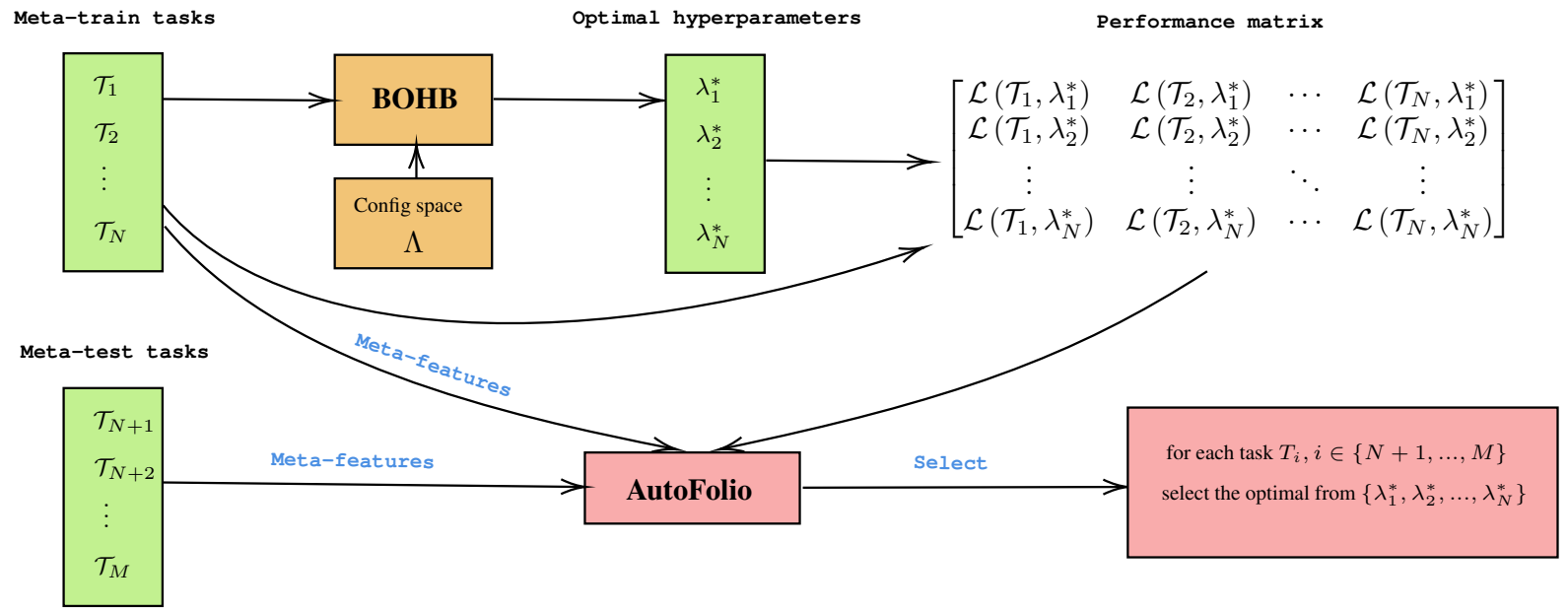

Fig. 5: Workflow of automl_freiburg. The approach first optimizes the hyperparameter configuration (including choices for training, input pipeline, and architecture) for every task (dataset) in our meta-training set using BOHB [33]. Afterwards, for each dataset $i$, the best found configuration $\lambda_{i}^{*}$ is evaluated on the other datasets $j \in\{1,2, \ldots, N\}, j \neq i$ to build the performance matrix (configurations $\times$ datasets). For training and configuring the meta-selection model based on performance matrix and the meta-features of the corresponding tasks, the approach uses AutoFolio [32]. At meta-test time, the model fitted by AutoFolio uses the meta-features of the test tasks in order to select a well-performing configuration.

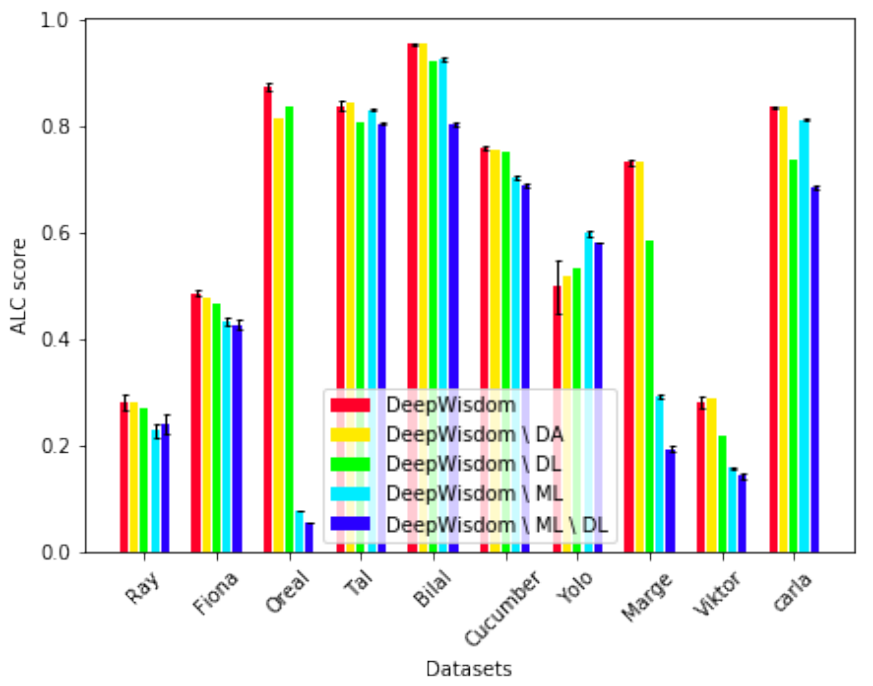

Fig. 6: Ablation study for DeepWisdom: We compare different versions of DeepWisdom's approach, with one component of their workflow disabled. "DeepWisdom \ML" represents DeepWisdom's original approach but with MetaLearning disabled. "DA" code for Data Augmentation and "DL" for Data Loading. The method variants are ordered by their average rank from left to right. Thus we observe that removing Data Augmentation does not make a lot of difference, while removing both Meta-Learning and Data Loading impacts the solution a lot. See Section 5.1.1 for details.
- Adaptive Strategies (AS): In this part, all adaptive parameter settings have been cancelled, such as the parameters settings according to the characteristics of data sets and the dynamic adjustments made during the training process. All relevant parameters are changed to default fixed values.

- Ensemble Learning (EL): In this part, all the parts of ensemble learning are removed. Instead of fusing the results of multiple models, the model that performs best in the validation set is directly selected for testing.

- Scoring Time Reduction (STR): In this part, all scoring time reduction settings were modified to default settings. Related parameters and data loading methods are same as those of baseline.

As it can be observed in Figure 7 the results of DeepBlueAI have been greatly improved compared with those of DeepBlueAI $\backslash$ AS $\backslash$ EL $\backslash$ STR (i.e., blue bar), indicating the effectiveness of the whole method. After removing the AS, the score of most data sets has decreased, indicating that adaptive strategies are better than fixed parameters or models, and has good generalization performance on different data sets. When STR is removed, the score of most data sets is reduced. Because the efficient data processing used can effectively reduce the scoring time, thereby improving the ALC score, which shows the effectiveness of the scoring time reduction. After EL is removed, the score of the vast majority of data sets has decreased, indicating the effectiveness of ensemble learning to improve the results.

\subsection{3 automl_freiburg}

According to the team automl freiburg, two of the most important components leading to the success of their approach are: meta-learning and hyperparameter optimization. For the ablation study, these components are removed or disabled in the following manner: 


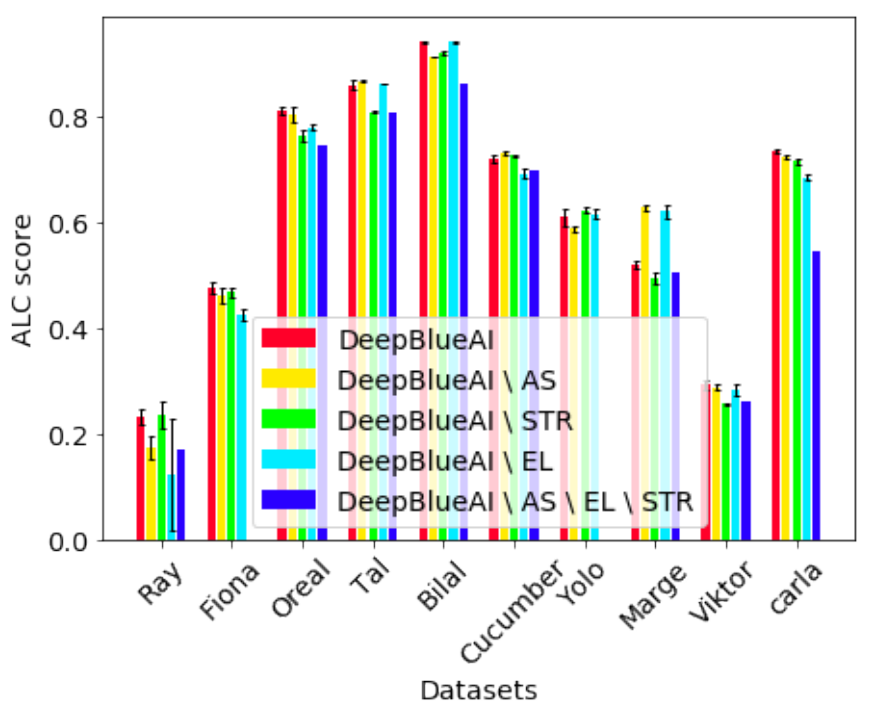

Fig. 7: Ablation study for DeepBlueAI: Comparison of different versions of DeepBlueAI's approach after removing some of the method's components. "DeepBlueAI \AS" represents their approach with Adaptive Strategy disabled. "EL" codes for Ensemble Learning and "STR" for Scoring Time Reduction. For each dataset, the methods are ordered by their average rank from left to right. While disabling each component separately yields moderate deterioration, disabling all of them yields a significant degradation in performance. See Section 5.1.2

- Meta-Learning with Random selector (MLR): This method randomly selects one configuration out of the set of most complementary configurations (Hammer, caltech_birds2010, cifar10, eurosat).

- Meta-Learning Generalist (MLG): This method does not use AutoFolio and always selects the generalist configuration that was optimized for the average improvement across all datasets.

- Hyperparameter Optimization (HPO): Instead of optimizing the hyperparameters of the metaselection model with AutoFolio, this method simply uses the default AutoFolio hyperparameters.

As previously mentioned, automl_freiburg focused on the computer vision domain (i.e., datasets Ray, Fiona, Cucumber, and Yolo). The results of their ablation study, shown in Figure 8 indicate that the hyperparameter search for the meta-model overfitted on the eight meta-train-datasets used (original vs HPO); eight datasets is generally regarded as insufficient in the realm of algorithm selection, but the team was limited by compute resources. However, the performance of the non-overfitted meta-model (HPO) clearly confirms the superiority of the approach over the random (MLR) and the generalist (MLG) baselines on all relevant datasets. More importantly, not only does this observation uncover further potential of automl freiburg's approach, it is also on par with the top two teams of the competition on these vision datasets: average rank 1.75 (automl_freiburg) versus 1.75 and 2.5 (DeepWisdom, DeepBlueAI). The authors emphasize that training the meta-learner on more than eight meta-train datasets could potentially lead to large improve-

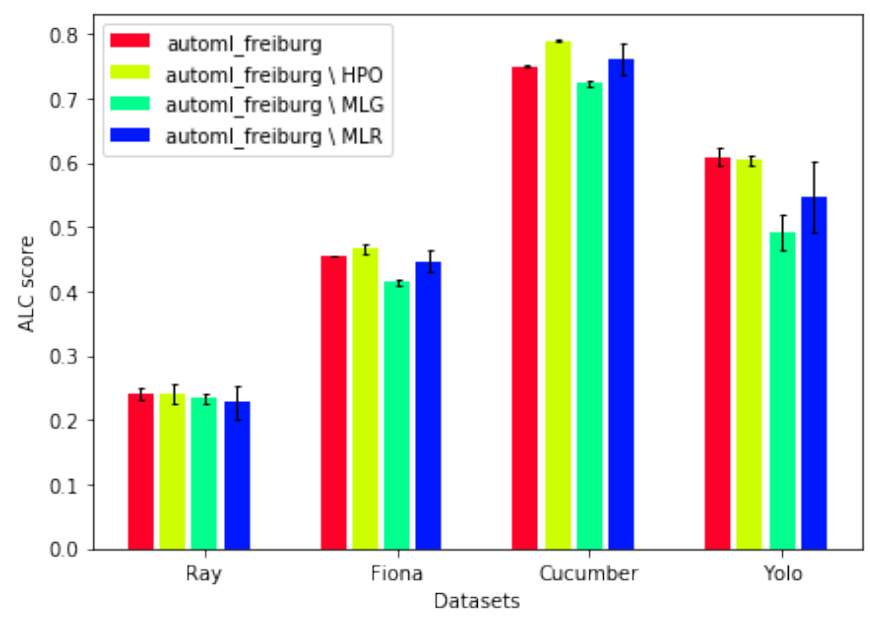

Fig. 8: Ablation study for automl_freiburg: Comparison of different versions of automl_freiburg's approach. Since the approach addresses only computer vision tasks, only results on image datasets (Ray, Cucumber) and video datasets (Fiona, Yolo) are shown. Average and error bars of ALC scores are computed over 9 runs. "automl_freiburg $\backslash$ HPO" represents automl_freiburg's approach with default AutoFolio hyperparameters. Likewise, "MLG" stands for the generalist configuration and "MLR" for randomly selecting a configuration from the pool of the most complementary configurations. See Section 5.1.3

ments in generalization performance. Despite the promising performance and outlook, results and conclusions should be interpreted conservatively due to the small number of metatest datasets relevant to automl_freiburg's approach.

\subsection{AutoML generalization ability of winning methods}

One crucial question for all AutoML methods is whether the method can have good performances on unseen datasets. If yes, we will say the method has AutoML generalization ability. To quantitatively measure this ability, we propose to compare the average rank of all top- 8 methods in both feedback phase and final phase, then compute the Pearson correlation (Pearson's $\rho$ ) of the 2 rank vectors (thus similar to Spearman's rank correlation [66]). Concretely, let $r_{X}$ be the average rank vector of top teams in feedback phase and $r_{Y}$ be that in final phase, then the Pearson correlation is computed by $\rho_{X, Y}=\operatorname{cov}\left(r_{X}, r_{Y}\right) / \sigma_{r_{X}} \sigma_{r_{Y}}$.

The average ranks of top methods are shown in Figure 9. with a Pearson correlation $\rho_{X, Y}=0.91$ and $p$-value $p=5.8 \times 10^{-4}$. This means that the correlation is statistically significant and no leaderboard overfitting is observed. Thus the winning solutions can indeed generalize to unseen datasets. Considering the diversity of final phase datasets and the arguably out-of-distribution final-test meta-features shown in Table 1 this is a feat from the AutoML community. Thus it's highly plausible that we are moving one step closer to a universal AutoML solution.

\subsection{Impact of $t_{0}$ in the ALC metric}

We recall that the Area under Learning Curve (ALC) is defined by 


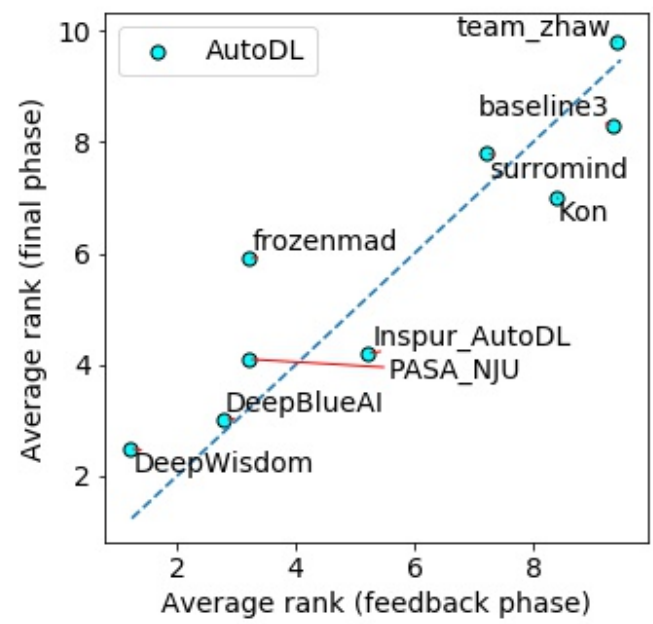

Fig. 9: Task over-modeling: We compare performance in the feed-back and final phase, in an effort to detect possible habituation to the feed-back datasets due to multiple submissions. The average rank of the top- 8 teams is shown. The figure suggests no strong over-modeling (over-fitting at the meta-learning level): A team having a significantly better rank in the feed-back phase than in the final phase would be over-modeling (far above the diagonal). The Pearson correlation is $\rho_{X, Y}=0.91$ and $p$-value $p=5.8 \times 10^{-4}$.

$$
\begin{aligned}
A L C & =\int_{0}^{1} s(t) d \tilde{t}(t) \\
& =\int_{0}^{T} s(t) \tilde{t}^{\prime}(t) d t \\
& =\frac{1}{\log \left(1+T / t_{0}\right)} \int_{0}^{T} \frac{s(t)}{t+t_{0}} d t
\end{aligned}
$$

where

$$
\tilde{t}(t)=\frac{\log \left(1+t / t_{0}\right)}{\log \left(1+T / t_{0}\right)}
$$

Thus $t_{0}$ parameterizes a weight distribution on the learning curve for computing the ALC. When $t_{0}$ is small, the importance weight at the beginning of the curve is large. Actually when $t_{0}$ varies from 0 to infinity, we have

$$
\lim _{t_{0} \rightarrow 0^{+}} A L C\left(t_{0}\right)=s(0)
$$

and

$$
\lim _{t_{0} \rightarrow+\infty} A L C\left(t_{0}\right)=\frac{1}{T} \int_{0}^{T} s(t) d t .
$$

So different $t_{0}$ might lead to different ALC ranking even if the learning curve $s(t)$ is fixed. It is then to be answered whether the choice of $t_{0}=60$ in AutoDL challenge is reasonable. For this, we reflect the impact of $t_{0}$ on the ALC scores and the final average ranking in Figure 10 Observation and discussion can be found in the caption. We conclude that $t_{0}$ does affect the ranking of ALC scores but the final ranking is robust to changes of $t_{0}$, justifying the choice of $t_{0}$ and the challenge setting.

\section{CONCLUSION AND FURTHER WORK}

In conclusion, we are encouraged to continue our challenge series in machine learning with code submission and blind testing in a well-defined identical computer environment, with a fixed time and memory budget. The latest one, the AutoDL challenge, helped pushing the state of the art in Automated Deep Learning. Our novel challenge design, with emphasis on "any-time learning", permitted to harvest answers to new questions.

Among other things, the challenge revealed that Automated Deep Learning methods are ripe for modalities such as image, video, speech, and text, but no unified solution emerged across modalities, and Deep Learning remained weaker than other methods for tabular data. This raises the question of developing new universal coding, generic workflows, or universal neural architectures. A step in this direction could be to organize a cross-modal Neural Architecture Search (NAS) challenge, to search for universal architectures. Intensive search in architecture space was impractical with the constrained time budget we provided for the AutoDL challenge, but with one order of magnitude more computational resources, it may be feasible.

Deep Learning methods have earned the reputation of being notoriously slow to train and require prohibitive computational resources in domains such as video processing. Not so anymore with "any-time learning methods" allowing users to stop training early and get reasonable performance. The wining teams succeeded in climbing the learning curve fast, without sacrificing the final performance. Transfer learning (fine tuning of pre-trained models), progressive increase in model complexity, fast data loading, and efficient exploration of data space, were key components to achieve these results.

The post-challenge analyses revealed the importance of meta-learning, through ablation studies conducted by winning teams. The teams demonstrated that generalizing to new unseen datasets is possible, and improves by metalearning, thus they effectively achieved a form of transfer learning. This calls for further research and we envision that a meta-learning challenge should be organized, to conduct a more controled study. Several settings have been proposed, including: (1) a challenge on model recommendation, similar to the movie recommendation Netflix challenge, in which a sparse matrix with just a few scores of models on a few datasets is initially provided and the goal is to find as quickly as possible the best performing model on a new dataset; (2) a challenge proposing training tasks and test tasks, aiming at training search agents capable of selecting the best performing models to solve the test tasks; (3) an on-line meta-learning challenge (or life-long-learning challenge) in which tasks are made available sequentially to models, who can retain some "memory" of past tasks to perform better in future tasks.

This challenge was limited to tensor data and multilabel problems. Other steps towards enlarging the scope of automated machine learning include generalizing to more complex data structures. This is partially addressed by the on-going AutoGraph challenge. Generalization to other types of tasks was addressed by the AutoSeries challenge. We intend to keep proposing more diverse types of data and 


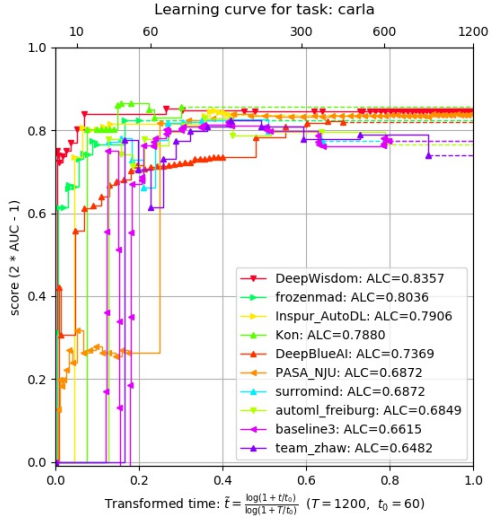

(a) Learning curves for the task Carla

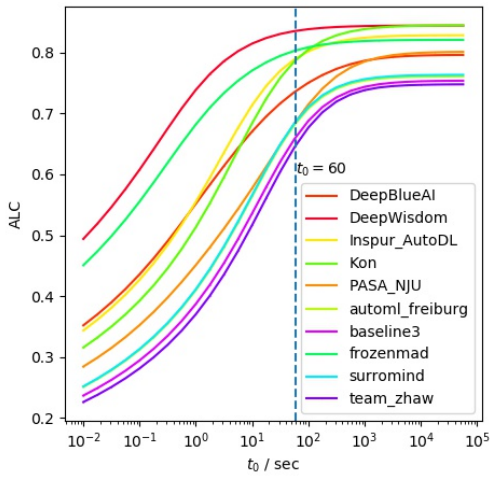

(b) Impact of $t_{0}$ on the ALC scores for task Carla.

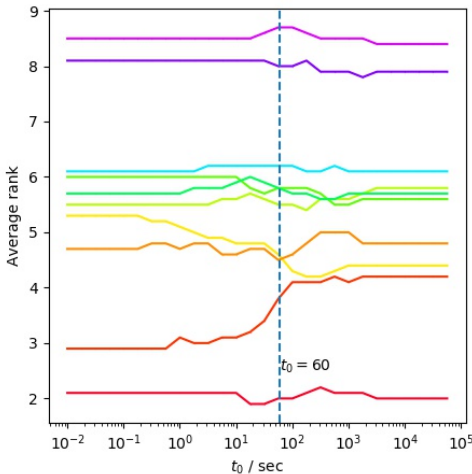

(c) Average rank among AutoDL final phase participants, using different $t_{0}$. The legend is hidden and is the same as that of Figure $10 \mathrm{~b}$

Fig. 10: Any-time learning vs. fixed-time learning: We evaluate the impact of parameter $t_{0}$ on the ALC scores and the final rank. This parameter allows us to smoothly adjust the importance of the beginning of the learning curve (and therefore the pressure imposed towards achieving any-time learning). When $\mathrm{t} 0$ is small, the ALC puts more emphasis on performances at the beginning of the learning curve and thus favors fast algorithms. When $t 0$ is large, similar weight is applied on the whole learning curve, performances are uniformly averaged, so being a little bit slow at the beginning is not that bad, and it is more important to have good final performance when the time budget is exhausted (fixed-time learning). The tabular dataset Carla is taken as example. The fact that two learning curves cross each other is a necessary condition for the impact of $t_{0}$ on their ranking on this task. Learning curves of top teams on this dataset are shown in 10a The impact of $t_{0}$ on the ALC scores of these curves is shown in $10 \mathrm{~b}$. We see that when $t_{0}$ changes, the ranking among participants can indeed change, typically the ALC of frozenmad is larger than that of Kon but this is not true for large $t_{0}$. In $10 \mathrm{c}$ the fact that the average rank (over all 10 final phase datasets) varies with $t_{0}$ also implies that $t_{0}$ can indeed affect the ranking of ALC on individual tasks. However, we see that the final ranking (i.e. that of average rank) is quite robust against changes of $t_{0}$. Very few exceptions exist such as PASA_NJU and Inspur_AutoDL. Overall, $t_{0}$ proved to have little impact, particularly on the ranking of the winners, which is another evidence that top ranking participants addressed well the any-time learning problem.

tasks to stimulate the community to make progress.

Lastly, challenges are meant to provide fair and reproducible evaluations removing the inventor-evaluator bias. However, other types of biases can crop up. One such bias stems from the choice of datasets. As organizers, we had to chose datasets with sufficient modeling difficulty to separate well the participants, yet not a too high intrinsic difficulty. By modeling difficulty, we mean the variance in performance between participants. By intrinsic difficulty we mean (1- the best attainable performance). Neither quantity being available to the organizers at the time of selection of the datasets, they must rely on the performances of the baseline methods to evaluate the difficulty of the tasks and thus the choice may be biased. Yet another type of bias is introduced by the baselime methods provided to the participants (such as Baseline 3 in this challenge).

Beyond research results, our challenges have a long lasting impact since we make available a large number of "public" datasets, and the code of winning solutions.

\section{ACKNOWLEDGMENTS}

This work was sponsored with a grant from Google Research (Zürich) and additional funding from 4Paradigm, Amazon and Microsoft. It has been partially supported by ICREA under the ICREA Academia programme. We also gratefully acknowledge the support of NVIDIA Corporation with the donation of the GPU used for this research. The team automl freiburg has partly been supported by the European Research Council (ERC) under the European Union's Horizon 2020 research and innovation programme under grant no. 716721. Further, automl_freiburg acknowledges Robert Bosch $\mathrm{GmbH}$ for financial support. It received in kind support from the institutions of the co-authors. We are very indebted to Olivier Bousquet and André Elisseeff at Google for their help with the design of the challenge and the countless hours that Andre spent engineering the data format. The special version of the CodaLab platform we used was implemented by Tyler Thomas, with the help of Eric Carmichael, CK Collab, LLC, USA. Many people contributed time to help formatting datasets, prepare baseline results, and facilitate the logistics. We are very grateful in particular to: Stephane Ayache (AMU, France), Hubert Jacob Banville (INRIA, France), Mahsa Behzadi (Google, Switzerland), Kristin Bennett (RPI, New York, USA), Hugo Jair Escalante (IANOE, Mexico and ChaLearn, USA), Gavin Cawley (U. East Anglia, UK), Baiyu Chen (UC Berkeley, USA), Albert Clapes i Sintes (U. Barcelona, Spain), Bram van Ginneken (Radboud U. Nijmegen, The Netherlands), Alexandre Gramfort (U. Paris-Saclay; INRIA, France), Yi-Qi Hu (4paradigm, China), Tatiana Merkulova (Google, Switzer- 
land), Shangeth Rajaa (BITS Pilani, India), Herilalaina Rakotoarison (U. Paris-Saclay, INRIA, France), Lukasz Romaszko (The University of Edinburgh, UK), Mehreen Saeed (FAST Nat. U. Lahore, Pakistan), Marc Schoenauer (U. ParisSaclay, INRIA, France), Michele Sebag (U. Paris-Saclay; CNRS, France), Danny Silver (Acadia University, Canada), Lisheng Sun (U. Paris-Saclay; UPSud, France), Wei-Wei Tu (4paradigm, China), Fengfu Li (4paradigm, China), Lichuan Xiang (4paradigm, China), Jun Wan (Chinese Academy of Sciences, China), Mengshuo Wang (4paradigm, China), Jingsong Wang (4paradigm, China), Ju Xu (4paradigm, China)

\section{REFERENCES}

[1] Z. Liu, Z. Xu, S. Rajaa, M. Madadi, J. Julio C. S. Jacques, S. Escalera, A. Pavao, S. Treguer, W.-W. Tu, and I. Guyon, "Towards Automated Deep Learning: Analysis of the AutoDL challenge series 2019," ser. Proceedings of Machine Learning Research, 2020.

[2] D. H. Wolpert and W. G. Macready, "No free lunch theorems for optimization," IEEE Transactions on Evolutionary Computation, vol. 1, no. 1, pp. 67-82, Apr. 1997. [Online]. Available: https://ti.arc.nasa.gov/m/profile/dhw/papers/78.pdf

[3] D. H. Wolpert, "The Lack of A Priori Distinctions Between Learning Algorithms," Neural Computation, vol. 8, no. 7, pp. 1341-1390, Oct. 1996. [Online]. Available: https://doi.org/10. 1162/neco.1996.8.7.1341

[4] D. Wolpert, "The Supervised Learning No-Free-Lunch Theorems," in Proceedings of the 6th Online World Conference on Soft Computing in Industrial Applications, Jan. 2001.

[5] I. Guyon, L. Sun-Hosoya, M. Boullé, H. J. Escalante, S. Escalera, Z. Liu, D. Jajetic, B. Ray, M. Saeed, M. Sebag, A. Statnikov, W.-W. $\mathrm{Tu}$, and E. Viegas, "Analysis of the AutoML Challenge series 20152018," in AutoML: Methods, Systems, Challenges, ser. The Springer Series on Challenges in Machine Learning, F. Hutter, L. Kotthoff, and J. Vanschoren, Eds. Springer Verlag, 2018. [Online]. Available: https://hal.archives-ouvertes.fr/hal-01906197

[6] B. Zoph and Q. V. Le, "Neural Architecture Search with Reinforcement Learning," arXiv:1611.01578 [cs], Nov. 2016, arXiv: 1611.01578. [Online]. Available: http://arxiv.org/abs/1611.01578

[7] M. Feurer, A. Klein, K. Eggensperger, J. Springenberg, M. Blum, and F. Hutter, "Efficient and Robust Automated Machine Learning," in Advances in Neural Information Processing Systems 28, C. Cortes, N. D. Lawrence, D. D. Lee, M. Sugiyama, and R. Garnett, Eds. Curran Associates, Inc., 2015, pp. 2962-2970. [Online]. Available: http://papers.nips.cc/paper/ 5872-efficient-and-robust-automated-machine-learning.pdt

[8] T. Elsken, J. H. Metzen, and F. Hutter, "Neural architecture search: A survey," J. Mach. Learn. Res., vol. 20, pp. 55:1-55:21, 2019.

[9] B. Baker, O. Gupta, N. Naik, and R. Raskar, "DESIGNING NEURAL NETWORK ARCHITECTURES USING REINFORCEMENT LEARNING," p. 18, 2017.

[10] R. Negrinho and G. Gordon, “DeepArchitect: Automatically Designing and Training Deep Architectures," arXiv:1704.08792 [cs, stat], Apr. 2017, arXiv: 1704.08792. [Online]. Available: http://arxiv.org/abs/1704.08792

[11] H. Cai, L. Zhu, and S. Han, "ProxylessNAS: Direct neural architecture search on target task and hardware," in International Conference on Learning Representations, 2019. [Online]. Available: https://openreview.net/forum?id=HylVB3AqYm

[12] H. Liu, K. Simonyan, and Y. Yang, "DARTS: differentiable architecture search," in 7th International Conference on Learning Representations, ICLR 2019, New Orleans, LA, USA, May 6-9, 2019. OpenReview.net, 2019.

[13] N. Fusi, R. Sheth, and M. Elibol, "Probabilistic matrix factorization for automated machine learning," in Proceedings of the 32nd International Conference on Neural Information Processing Systems, ser. NIPS'18. Red Hook, NY, USA: Curran Associates Inc., 2018, p. 3352-3361.

[14] C. Cortes, X. Gonzalvo, V. Kuznetsov, M. Mohri, and S. Yang, "AdaNet: Adaptive structural learning of artificial neural networks," in Proceedings of the 34th International Conference on Machine Learning, ser. Proceedings of Machine Learning Research, D. Precup and Y. W. Teh, Eds., vol. 70. International Convention Centre, Sydney, Australia: PMLR, 06-11 Aug 2017, pp. 874-883.
[Online]. Available: http://proceedings.mlr.press/v70/cortes17a. html

[15] E. Real, S. Moore, A. Selle, S. Saxena, Y. L. Suematsu, J. Tan, Q. V. Le, and A. Kurakin, "Large-scale evolution of image classifiers," in Proceedings of the 34th International Conference on Machine Learning - Volume 70, ser. ICML'17. JMLR.org, 2017, p. 2902-2911.

[16] H. Pham, M. Guan, B. Zoph, Q. Le, and J. Dean, "Efficient neural architecture search via parameters sharing," in Proceedings of the 35th International Conference on Machine Learning, ser. Proceedings of Machine Learning Research, J. Dy and A. Krause, Eds., vol. 80. Stockholmsmässan, Stockholm Sweden: PMLR 10-15 Jul 2018, pp. 4095-4104. [Online]. Available: http: //proceedings.mlr.press/v80/pham18a.html

[17] E. Real, C. Liang, D. R. So, and Q. V. Le, "AutoML-Zero: Evolving Machine Learning Algorithms From Scratch," arXiv:2003.03384 [cs, stat], Mar. 2020, arXiv: 2003.03384. [Online]. Available: http://arxiv.org/abs/2003.03384

[18] C. Finn, P. Abbeel, and S. Levine, "Model-agnostic meta-learning for fast adaptation of deep networks," in Proceedings of the 34th International Conference on Machine Learning, ser. Proceedings of Machine Learning Research, D. Precup and Y. W. Teh, Eds., vol. 70. International Convention Centre, Sydney, Australia: PMLR, 06-11 Aug 2017, pp. 1126-1135. [Online]. Available: http://proceedings.mlr.press/v70/finn17a.html

[19] C. Finn, A. Rajeswaran, S. Kakade, and S. Levine, "Online meta-learning," in Proceedings of the 36th International Conference on Machine Learning, ser. Proceedings of Machine Learning Research, K. Chaudhuri and R. Salakhutdinov, Eds., vol. 97. Long Beach, California, USA: PMLR, 09-15 Jun 2019, pp. 1920-1930. [Online]. Available: http://proceedings.mlr.press/v97/finn19a.html

[20] A. Yang, P. M. Esperança, and F. M. Carlucci, "Nas evaluation is frustratingly hard," in International Conference on Learning Representations, 2020. [Online]. Available: https: // openreview.net/forum?id=HygrdpVKvr

[21] Z. Liu, Z. Xu, S. Escalera, I. Guyon, J. J. Junior, M. Madadi, A. Pavao, S. Treguer, and W.-W. Tu, "Towards Automated Computer Vision: Analysis of the AutoCV Challenges 2019," Nov. 2019. [Online]. Available: https://hal.archives-ouvertes.fr/ hal-02386805

[22] S. Lim, I. Kim, T. Kim, C. Kim, and S. Kim, "Fast autoaugment," in Advances in Neural Information Processing Systems 32, H. Wallach, H. Larochelle, A. Beygelzimer, F. dAlché-Buc, E. Fox, and R. Garnett, Eds. Curran Associates, Inc., 2019, pp. 6665-6675. [Online]. Available: http://papers.nips.cc/paper/ 8892-fast-autoaugment.pdf

[23] E. D. Cubuk, B. Zoph, D. Mané, V. Vasudevan, and Q. V. Le, "Autoaugment: Learning augmentation strategies from data," in 2019 IEEE/CVF Conference on Computer Vision and Pattern Recognition (CVPR), 2019, pp. 113-123.

[24] K. He, X. Zhang, S. Ren, and J. Sun, “Deep residual learning for image recognition," in 2016 IEEE Conference on Computer Vision and Pattern Recognition (CVPR), 2016, pp. 770-778.

[25] R.-E. Fan, K.-W. Chang, C.-J. Hsieh, X.-R. Wang, and C.-J. Lin, "LIBLINEAR: A Library for Large Linear Classification," p. 31.

[26] S. Hochreiter and J. Schmidhuber, "Long short-term memory," Neural computation, vol. 9, no. 8, pp. 1735-1780, 1997.

[27] J. Devlin, M.-W. Chang, K. Lee, and K. Toutanova, "BERT: Pre-training of deep bidirectional transformers for language understanding," in Proceedings of the 2019 Conference of the North American Chapter of the Association for Computational Linguistics: Human Language Technologies, Volume 1 (Long and Short Papers). Minneapolis, Minnesota: Association for Computational Linguistics, Jun. 2019, pp. 4171-4186. [Online]. Available: https://www.aclweb.org/anthology/N19-1423

[28] S. Davis and P. Mermelstein, "Comparison of parametric representations for monosyllabic word recognition in continuously spoken sentences," IEEE transactions on acoustics, speech, and signal processing, vol. 28, no. 4, pp. 357-366, 1980.

[29] D. G. Lowe, "Distinctive image features from scale-invariant keypoints," Int. J. Comput. Vision, vol. 60, no. 2, p. 91-110, Nov. 2004. [Online]. Available: https://doi.org/10.1023/B:VISI. 0000029664.99615 .94

[30] J. S. Chung, A. Nagrani, and A. Zisserman, "Voxceleb2: Deep speaker recognition," in INTERSPEECH, 2018.

[31] P. Vincent, H. Larochelle, Y. Bengio, and P.-A. Manzagol, "Extracting and composing robust features with denoising autoencoders," 
in Proceedings of the 25th international conference on Machine learning, 2008, pp. 1096-1103.

[32] M. Lindauer, H. H. Hoos, F. Hutter, and T. Schaub, "AutoFolio: an automatically configured algorithm selector," Journal of Artificial Intelligence Research, vol. 53, no. 1, pp. 745-778, May 2015.

[33] S. Falkner, A. Klein, and F. Hutter, "BOHB: Robust and Efficient Hyperparameter Optimization at Scale," p. 10.

[34] F. Hutter, H. H. Hoos, and K. Leyton-Brown, "Sequential modelbased optimization for general algorithm configuration," in International conference on learning and intelligent optimization. Springer, 2011, pp. 507-523.

[35] J. S. Bergstra, R. Bardenet, Y. Bengio, and B. Kégl, "Algorithms for Hyper-Parameter Optimization," in Advances in Neural Information Processing Systems 24, J. Shawe-Taylor, R. S. Zemel, P. L. Bartlett, F. Pereira, and K. Q. Weinberger, Eds. Curran Associates, Inc., 2011, pp. 2546-2554. [Online]. Available: http://papers.nips.cc/ paper/4443-algorithms-for-hyper-parameter-optimization.pdf

[36] O. Russakovsky, J. Deng, H. Su, J. Krause, S. Satheesh, S. Ma, Z. Huang, A. Karpathy, A. Khosla, M. Bernstein, A. C. Berg, and L. Fei-Fei, "ImageNet Large Scale Visual Recognition Challenge," arXiv:1409.0575 [cs], Jan. 2015, arXiv: 1409.0575. [Online]. Available: http://arxiv.org/abs/1409.0575

[37] R. Caruana, A. Niculescu-Mizil, G. Crew, and A. Ksikes, "Ensemble selection from libraries of models," in Twentyfirst international conference on Machine learning - ICML '04. Banff, Alberta, Canada: ACM Press, 2004, p. 18. [Online]. Available: http://portal.acm.org/citation.cfm?doid=1015330.1015432

[38] G. Ke, Q. Meng, T. Finley, T. Wang, W. Chen, W. Ma, Q. Ye, and T.-Y. Liu, "LightGBM: A Highly Efficient Gradient Boosting Decision Tree," in Advances in Neural Information Processing Systems 30, I. Guyon, U. V. Luxburg, S. Bengio, H. Wallach, R. Fergus, S. Vishwanathan, and R. Garnett, Eds. Curran Associates, Inc., 2017, pp. 3146-3154. [Online]. Available: http://papers.nips.cc/paper/ 6907-lightgbm-a-highly-efficient-gradient-boosting-decision-tree. pdf

[39] D. Tran, H. Wang, L. Torresani, J. Ray, Y. LeCun, and M. Paluri, “A closer look at spatiotemporal convolutions for action recognition," in Proceedings of the IEEE conference on Computer Vision and Pattern Recognition, 2018, pp. 6450-6459.

[40] Y. Kim, "Convolutional neural networks for sentence classification," in Proceedings of the 2014 Conference on Empirical Methods in Natural Language Processing (EMNLP). Doha, Qatar: Association for Computational Linguistics, Oct. 2014, pp. 1746-1751. [Online]. Available: https://www.aclweb.org/anthology/D14-1181

[41] R. Girshick, J. Donahue, T. Darrell, and J. Malik, "Rich feature hierarchies for accurate object detection and semantic segmentation," in The IEEE Conference on Computer Vision and Pattern Recognition (CVPR), June 2014

[42] S. Lim, I. Kim, T. Kim, C. Kim, and S. Kim, "Fast autoaugment," in Advances in Neural Information Processing Systems, 2019, pp. 6662 6672.

[43] J. S. Bridle and M. D. Brown, "An experimental automatic word recognition system," JSRU Report, vol. 1003, no. 5, p. 33, 1974.

[44] K. Cho, B. van Merriënboer, C. Gulcehre, D. Bahdanau, F. Bougares, H. Schwenk, and Y. Bengio, "Learning phrase representations using RNN encoder-decoder for statistical machine translation," in Proceedings of the 2014 Conference on Empirical Methods in Natural Language Processing (EMNLP). Doha, Qatar: Association for Computational Linguistics, Oct. 2014, pp. 1724-1734. [Online]. Available: https://www.aclweb.org/ anthology/D14-1179

[45] A. Joulin, E. Grave, P. Bojanowski, and T. Mikolov, "Bag of tricks for efficient text classification," in Proceedings of the 15th Conference of the European Chapter of the Association for Computational Linguistics: Volume 2, Short Papers. Valencia, Spain: Association for Computational Linguistics, Apr. 2017, pp. 427-431. [Online]. Available: https://www.aclweb.org/anthology/E17-2068

[46] J. Hu, L. Shen, and G. Sun, "Squeeze-and-excitation networks," in The IEEE Conference on Computer Vision and Pattern Recognition (CVPR), June 2018.

[47] X. Chen, Q. Lin, and ..., "Neural feature search: A neural architecture for automated feature engineering," in ICDM'19, October 2019.

[48] J. Bergstra, D. Yamins, and D. D. Cox, "Making a science of model search: Hyperparameter optimization in hundreds of dimensions for vision architectures," in Proceedings of the 30th International
Conference on International Conference on Machine Learning - Volume 28, ser. ICML'13. JMLR.org, 2013, p. I-115-I-123.

[49] K. Brain, "AutoCLINT, Automatic Computationally LIght Network Transfer," https://github.com/kakaobrain/autoclint 2019.

[50] L. Xu, F. Hutter, J. Shen, H. Hoos, and K. Leyton-Brown, "SATzilla2012: Improved algorithm selection based on costsensitive classification models," Proceedings of SAT Challenge 2012: Solver and Benchmark Descriptions, pp. 55-58, Jan. 2012.

[51] M. Tan and Q. Le, "EfficientNet: Rethinking model scaling for convolutional neural networks," in Proceedings of the 36th International Conference on Machine Learning, ser. Proceedings of Machine Learning Research, K. Chaudhuri and R. Salakhutdinov, Eds., vol. 97. Long Beach, California, USA: PMLR, 09-15 Jun 2019, pp. 6105-6114. [Online]. Available: http://proceedings.mlr. press/v97/tan19a.html

[52] D. P. Kingma and J. Ba, "Adam: A Method for Stochastic Optimization," arXiv:1412.6980 [cs], Dec. 2014, arXiv: 1412.6980. [Online]. Available: http://arxiv.org/abs/1412.6980

[53] I. Loshchilov and F. Hutter, "Decoupled weight decay regularization," in 7th International Conference on Learning Representations, ICLR 2019, New Orleans, LA, USA, May 6-9, 2019. OpenReview.net, 2019. [Online]. Available: https://openreview.net/forum?id=Bkg6RiCqY7

[54] Y. Nesterov, "A method for unconstrained convex minimization problem with the rate of convergence o $\left(1 / \mathrm{k}^{\wedge} 2\right),{ }^{\prime}$ in Doklady an ussr, vol. 269, 1983, pp. 543-547.

[55] I. Loshchilov and F. Hutter, "SGDR: stochastic gradient descent with warm restarts," in 5th International Conference on Learning Representations, ICLR 2017, Toulon, France, April 24-26, 2017, Conference Track Proceedings, 2017.

[56] A. Krizhevsky, G. Hinton et al., "Learning multiple layers of features from tiny images," 2009.

[57] "The ham 10000 dataset, a large collection of multi-source dermatoscopic images of common pigmented skin lesions," Scientific Data, vol. 5, no. 1, p. 180161, 2018.

[58] Y. LeCun, C. Cortes, and C. Burges, "Mnist handwritten digit database," ATT Labs [Online]. Available: http://yann.lecun.com/exdb/mnist, vol. 2, 2010.

[59] P. Welinder, S. Branson, T. Mita, C. Wah, F. Schroff, S. Belongie, and P. Perona, "Caltech-UCSD Birds 200," California Institute of Technology, Tech. Rep. CNS-TR-2010-001, 2010.

[60] J. N. Kather, C.-A. Weis, F. Bianconi, S. M. Melchers, L. R. Schad, T. Gaiser, A. Marx, and F. G. Z"ollner, "Multi-class texture analysis in colorectal cancer histology," Scientific reports, vol. 6, p. 27988, 2016.

[61] P. Helber, B. Bischke, A. Dengel, and D. Borth, "Eurosat: A novel dataset and deep learning benchmark for land use and land cover classification," 2017.

[62] L. Xu, H. Hoos, and K. Leyton-Brown, "Hydra: Automatically configuring algorithms for portfolio-based selection," pp. 210-216.

[63] M. Feurer, K. Eggensperger, S. Falkner, M. Lindauer, and F. Hutter, "Practical automated machine learning for the automl challenge 2018," in AutoML workshop at international conference on machine learning (ICML), 2018.

[64] L. Xu, F. Hutter, H. Hoos, and K. Leyton-Brown, "Hydra-MIP: Automated algorithm configuration and selection for mixed integer programming," in Proc. of RCRA workshop at IJCAI, 2011.

[65] F. Hutter, H. Hoos, and K. Leyton-Brown, "Sequential modelbased optimization for general algorithm configuration," pp. 507523.

[66] "Spearman's rank correlation coefficient," Apr. 2020, page Version ID: 953109044 . [Online]. Available: https://en.wikipedia.org/w/index.php?title=Spearman $\%$ 27s rank correlation coefficient\&oldid $=953109044$

Zhengying Liu is a PhD student at Université Paris-Saclay, under the supervision of Isabelle Guyon. He received his bachelor degree at Peking University in fundamental mathematics and physics (double major) in 2013, master's degree in mathematics and computer science (double major) at Ecole polytechnique in 2017. His research interests lie in AutoML, deep learning and artificial intelligence in general including logic and automatic mathematical reasoning. He is one of the organizers of AutoDL challenges and has organized corresponding workshops at ECMLPKDD 2019 and NeurIPS 2019. 
Adrien Pavao is a PhD student at Universite Paris-Saclay, under the supervision of Isabelle Guyon. He received his master's degree in computer science and machine learning at Université Paris-Saclay in 2019. His research topics include the methodology and experimental design in machine learning, the performance comparison between models and the organization of competitions.

Zhen $\mathbf{X U}$ is a Machine Learning Engineer at 4Paradigm, China. He works in Automated Machine Learning (AutoML) with applications in time series, image, text, speech, graph, etc. He also takes an active role in organizing AutoML/AutoDL challenges in top conferences, e.g. AutoCV, AutoSpeech, AutoSeries. Zhen received Engineering Degree (Diplôme d'Ingénieur) from Ecole polytechnique, Paris, majoring in Computer Science. He has a double diploma of Master in Applied Mathematics from University Paris-Sud, Orsay.

Sergio Escalera is Full Professor at Universitat de Barcelona and member of the Computer Vision Center at UAB. He leads the Human Behavior Analysis Group at UB and Computer Vision Center. He is series editor of The Springer Series on Challenges in Machine Learning. He is vice-president of ChaLearn Challenges in Machine Learning, leading ChaLearn Looking at People events. He is also member of the European Laboratory for Learning and Intelligent Systems. His research interests include automatic deep learning and analysis of humans from visual and multi-modal data, with special interest in inclusive, transparent, and fair affective computing and people characterization: personality and psychological profile computing.

Isabelle Guyon is Full Professor of Data Science and Machine Learning at Université Paris-Saclay, head master of the CS Artifical Intelligence master program, and researcher at INRIA. She is also founder and president of ChaLearn, a non-profit dedicated to organizing challenges in Machine Learning and community lead on the development of the competition platform CodaLab. She was co-program chair of NeurIPS 2016 and co-general chair of NeurIPS 2017, and now serving on the board of NeurIPS. She is an AMIA and an ELLIS fellow and action editor at JMLR, CiML springer series editor, and BBVA award recipient.

Julio C. S. Jacques Junior is a postdoctoral researcher at the Computer Science, Multimedia and Telecommunications department at Universitat Oberta de Catalunya (UOC), within the Scene Understanding and Artificial Intelligence (SUNAI) group. He also collaborates within the Computer Vision Center (CVC) and Human Pose Recovery and Behavior Analysis (HUPBA) group at Universitat Autonoma de Barcelona (UAB) and University of Barcelona (UB), as well as within ChaLearn Looking at People.

Meysam Madadi obtained his MS degree and $\mathrm{PhD}$ in Computer Vision at the Universitat Autònoma de Barcelona (UAB) in 2013 and 2017, respectively. He is currently a postdoc researcher at Computer Vision Center (CVC), UAB. He has been a member of Human Pose Recovery and Behavior Analysis (HUPBA) group since 2012. His main interest is deep learning, computer vision, human pose estimation and garment modeling. automl_freiburg The automl_freiburg team at the University of Freiburg (and nowadays also at the Leibniz University Hannover; Germany) was founded in 2015 and won several tracks of the first and second AutoML challenge. Members of the current challenge team are Fabio Ferreira, Danny Stoll, Arber Zela, Thomas Nierhoff, Prof. Marius Lindauer and Prof. Frank Hutter. Alumni of the challenge team include Matthias Feurer, Katharina Eggensperger, Aaron Klein and Stefan Falkner. Besides publications on AutoML at top journals and conferences, the group is well known for their open-source AutoML tools, such as Auto-Sklearn and Auto-PyTorch, see www.automl.org

DeepBlueAl Team leader Zhipeng Luo received the M.S. degree from Peking University. He has nearly 6 years of machine learning experience. He has rich practical experience in computer vision, data mining and natural language processing. He has won championships in many top conference competitions, including CVPR, ICCV, KDD, NerullPS, SIGIR, ACM MM , WSDM, CIKM, PAKDD, IEEE ISI. Members of the DeepBlueAl team are Chunguang Pan, Ge Li, Jin Wang and Kangning Niu.

DeepWisdom is a joint team of DeepWisdom and Xiamen University under the guidance of Prof. Rongrong $\mathrm{Ji}$ and Chenglin Wu. Prof. Ji is currently a Professor and the Director of the Intelligent Multimedia Technology Laboratory, and the Dean Assistant with the School of Information Science and Engineering, Xiamen University, Xiamen, China, with over 100 papers published in international journals and conferences. Chenglin Wu is CEO of DeepWisdom. Other members of the team are Yang Zhang, Huixia Li, Sirui Hong and Youcheng Xiong. DeepWisdom is to build Al with Al, see http://fuzhi.ai// 